\title{
3 Ansätze einer Neuklassifikation
}

\subsection{Linguistische Kategorienbildung}

So wie die Dinge der Welt im Prinzip problemlos in unterschiedliche Kategorien eingeteilt werden können, stellt auch die Kategorisierung sprachlicher Phänomene im Grundsatz keine besonders schwierige Aufgabe dar: Um eine Klasse aufzustellen, ist ein klassenkonstituierendes Merkmal bzw. Merkmalbündel auszuwählen und alle in Frage kommenden Phänomene sind darauf hin zu prüfen, ob sie diesem Merkmal bzw. Merkmalset entsprechen oder nicht. Dass dabei Prototypikalitätseffekte auftreten und es einige Kategorienvertreter gibt, deren Klassenzuordnung Probleme bereitet, versteht sich von selbst. Die Schwierigkeit bei der Klassifikation liegt weniger in der Etablierung der Kategorien selbst als in deren Relevanz. Es ist z. B. leicht, eine Kategorie für alle Farbadjektive, die auf - $a$ enden und aus dem Lateinischen stammen oder für alle Verben, die stark flektieren und ein Bewegungsereignis bezeichnen, aufzustellen. Ob diese Kategorien freilich sinnvoll sind, ob sie das Funktionieren von Sprache besser verständlich machen können als andere kategorielle Einteilungen, ist die entscheidende Frage.

Den Eindruck einer ad-hoc gebildeten sprachwissenschaftlichen Kategorie erwecken auch manche der im vorangegangenen Kapitel besprochenen Definitionen der Klasse FVG. Wenn etwa Heine (2006: 66f., 74f.) für eine „Kerngruppe“ von FVG u. a. die Kriterien einführt, dass a) das Verb „,in seiner (ursprünglichen) Hauptbedeutung den Feldern ,Bewegung“ oder ,Besitzwechsel““ entstammt, b) „das Substantiv zu den Valenz tragenden Nomina“ gehört und es c) weder pronominalisierbar noch erfragbar sein darf, wirft dies die Frage auf, weshalb FVG gerade nach diesem Kriterienkatalog definiert sind: Weshalb geht die Definition gerade von Verben der Bewegung und des Besitzwechsels aus, warum nicht auch von Verben der Position (stehen) oder des Besitzes (haben)? Warum sollte genau diese Kombination lexikalischer Restriktionen für die V-Position und syntaktischer Restriktionen für die N-Position ein Set von Eigenschaften bilden, das eine bestimmte Gruppe von Nomen-Verb-Verbindungen signifikant von anderen solcher Verbindungen abhebt? Hier wird erkennbar versucht, einen durch die ältere Forschungsliteratur mehr oder weniger fest vorgegebenen sprachwissenschaftlichen Gegenstand nachträglich mit schärferen Konturen zu versehen.

Wenn auch in der vorliegenden Studie der Versuch unternommen wird, eine Klasse FVG zu definieren und hinsichtlich ihrer semantischen und syntaktischen Eigenschaften zu beschreiben, so geht es nicht darum, einen durch die Tradition

Ә Open Access. (c) 2021 Volker Harm, publiziert von De Gruyter. (c) BY Dieses Werk ist lizenziert unter der Creative Commons Attribution-NonCommercial-NoDerivatives 4.0 Lizenz.

https://doi.org/10.1515/9783110661255-003 
vorgegebenen und im Laufe der jüngeren Forschungsdiskussion fragwürdig gewordenen Gegenstand in irgendeiner Weise zu rechtfertigen oder zu ,retten'. Ziel ist vielmehr zu zeigen, dass es notwendig ist, innerhalb der Nomen-Verb-Verbindungen eine besondere Klasse von Verbindungen zu identifizieren, weil diese in zentralen Merkmalen nicht mit anderen Nomen-Verb-Verbindungen übereinstimmt. Diese besondere Klasse ist zwar in weiten Teilen mit dem Kernbestand der bisher als FVG beschriebenen Klasse von N+V-Fügungen identisch - daher wird auch der Terminus beibehalten -, sie wird hier jedoch auf gänzlich andere Weise konstituiert als die vorwiegend nach der Funktion bestimmte herkömmliche Klasse der FVG. Der wesentliche Unterschied besteht, wie erwähnt, darin, dass der Fokus nicht auf dem Verb, sondern auf der Nomen-Verb-Konstruktion als Ganzer liegt (wobei ,Konstruktion‘ vorläufig noch informell im Sinne von,Verbindung“/,Kombination` verwendet werden soll; Genaueres bietet Kapitel 4.3).

Die klassendefinierende konstruktionelle Eigenschaft, auf die es hier ankommt, ist in dem besonderen Bedeutungsaufbau der $\mathrm{N}+\mathrm{V}$-Verbindung zu sehen, und zwar in dem Verhältnis zwischen der Gesamtbedeutung der N+V-Konstruktion und den Bedeutungen ihrer Teile. Im Unterschied $\mathrm{zu}$ allen anderen $\mathrm{N}+\mathrm{V}$-Verbindungen zeichnet sich der Bedeutungsaufbau der hier als ,FVG' etikettierten Klasse von Konstruktionen dadurch aus, dass die lexikalische Bedeutung des Nomens die lexikalische Gesamtbedeutung der Konstruktion impliziert.

Im Folgenden gilt es zunächst, diese Implikationsrelation zwischen der Nominal- und der Konstruktionsbedeutung näher zu bestimmen und vor allem auch das Besondere an dieser Relation herauszustellen. Denn diese Relation ist nur dann als aussagekräftiges Kriterium für die Abgrenzung einer Klasse verwendbar, wenn damit eine fundamentale Unterscheidung gegenüber anderen NomenVerb-Kombinationen getroffen ist.

\subsection{Implikationsrelationen innerhalb der N+V-Konstruktion}

\subsubsection{Die Implikationsrelation}

In der Lexikologie stellt die Implikationsrelation die wichtigste Relation zwischen Wortbedeutungen dar, da sie bei der Ermittlung anderer semantischer Relationen (Hyponymie/Hyperonymie, Synonymie, Gegensatzrelationen) eine zentrale Rolle spielt (vgl. Cann 2002: 553; Cruse 2002: 543). Strenggenommen sind Implikationsrelationen jedoch nicht für Wörter, sondern für Sätze oder, genauer gesagt, für Aussagen definiert. So liegt nach der klassischen Formulierung von Lyons unter der folgenden Bedingung eine Implikation zwischen zwei Sätzen bzw. Aussagen vor: 
Man sagt, daß ein Satz $S_{1}$ einen anderen Satz $S_{2}$ impliziert [...], wenn Sprecher der Sprache darin übereinstimmen, daß es nicht möglich ist, $S_{1}$ explizit zu behaupten und $S_{2}$ explizit zu verneinen (Lyons 1971: 455f.).

Etwas vereinfacht und besser handhabbar ist diese Definition in der folgenden Umformulierung:

Ein Satz $S_{1}$ impliziert einen anderen Satz $S_{2}$, wenn Sprecher der Sprache darin übereinstimmen, dass, wenn $S_{1}$ der Fall ist, auch $S_{2}$ der Fall ist.

Wenn nach semantischen Relationen zwischen Wortbedeutungen gefragt wird, hat es sich seit Lyons eingebürgert, Relationen zwischen Wortbedeutungen über Relationen zwischen Sätzen zu bestimmen, welche die betreffenden Wörter enthalten (vgl. auch Cruse 1986: 14; 2002: 543). So kann z. B. eine Implikationsrelation zwischen den Sätzen Hans schleicht und Hans bewegt sich fort als heuristisches Mittel dafür genutzt werden, um auch für die Verben schleichen und sich fortbewegen bzw. genauer gesagt die Wortbedeutungen ,schleichen' und ,sich fortbewegen' eine Implikationsrelation plausibel zu machen. Gleiches gilt nicht nur für Verben, sondern grundsätzlich auch für Substantive (z. B. für Hund und Tier in Sätzen wie der Hund wedelt mit dem Schwanz und Das Tier wedelt mit dem Schwanz). Eine über die Implikation von Satzaussagen ermittelte Implikation zwischen Wortbedeutungen wird auch als ,lexikalische Implikation' bezeichnet (vgl. „lexical entailment“, Miller/Fellbaum 1992: 219).

Wenn die Implikation als eine Relation zwischen Wortbedeutungen zu beschreiben ist, wirft dies die Frage auf, was Wortbedeutungen denn eigentlich seien. Diese Frage kann hier nicht beantwortet werden, vor allem nicht angesichts der zahlreichen, $z$. T. fundamental divergierenden Theorien zur Wortbedeutung (vgl. dazu den Überblick in Geeraerts 2010). Rezente Ansätze zur lexikalischen Semantik - die Conceptual Semantics (Jackendoff 2002), Natural Semantic Metalanguage (Wierzbicka 1996), Generative Lexicon (Pustejovsky 1995) sowie die Kognitive Semantik (Croft/Cruse 2004) - stimmen bei allen Unterschieden aber immerhin in der zentralen Annahme überein, dass Wortbedeutungen grundsätzlich konzeptueller Natur sind, also im Kern als kognitive Einheiten, eben ,Konzepte', beschrieben werden müssen. ${ }^{49}$ Konzepte kann man als mentale Repräsentationen von Dingen in der Welt oder auch Eigenschaften von Dingen der Welt ansehen (vgl. Murphy 2002: 270). Diese Repräsentationen fassen typi-

49 Zum Status des Konzepts in den genannten Beschreibungsansätzen vgl. auch Murphy (2010: 58-77). 
scherweise Einzelnes zu Klassen zusammen. Sie können entweder als Gegenstands- oder als Eigenschaftskonzepte realisiert sein. Eigenschaftskonzepte können dabei auch eine Spezifikation hinsichtlich ihrer zeitlichen Geltung aufweisen; in diesem Fall spricht man von Ereigniskonzepten (zu Ereignissen als Eigenschaften s. Fabricius-Hansen 1991: 692f.; Engelberg 2000: 231). Ereigniskonzepte wiederum sind in statische und nicht-statische Ereignisse $\mathrm{zu}$ unterteilen (vgl. etwa den Gegensatz zwischen STEHEN vs. LAUFEN).

Wenn einem Konzept per Sprachkonvention ein Ausdruck - typischerweise ein Wort - zugeordnet ist, spricht man von einem lexikalisierten Konzept; Konzepte müssen aber auch nicht zwingend durch einen eigenen Ausdruck repräsentiert sein (z. B. das Konzept KEINEN DURST MEHR HABEND, dem kein eigenes Wort entspricht). Die Lexikalisierung von Konzepten folgt bestimmten Mustern: Gegenstandskonzepte werden als Substantive, nicht temporal spezifizierte Eigenschaftskonzepte als Adjektive und Ereigniskonzepte als Verben kodiert (vgl. Langacker 1991: 15, 19). Bei diesen Zuordnungen handelt es sich allerdings nicht um zwingend gegebene, sondern lediglich um prototypische Lexikalisierungen (Figge 1993: 124; Murphy 2010: 139-142). So können insbesondere auch Ereigniskonzepte als Substantive lexikalisiert sein, und zwar etwa im Fall von Nomina wie Aufführung oder Sturz. Dass hier in den entsprechenden Lesarten nicht Gegenstandskonzepte, sondern tatsächlich Ereigniskonzepte vorliegen, ist dadurch erwiesen, dass hier zum einen temporale Attribuierungen wie die gestrige Aufführung oder der gestrige Sturz möglich sind und zum anderen dass beide Ausdrücke relational sind, d. h. nur mit Bezug auf einen (oder mehrere) Partizipanten definiert werden können: die Aufführung des Regisseurs/des Stücks, der Sturz des Kindes.

Eine grundsätzliche Trennung des konzeptuellen Gehalts, den ein Wort ausdrückt, von seiner Wortartenzugehörigkeit wird auch in der von Ackerman/Webelhuth (1998: 6f.) vorgelegten Theorie des Prädikats vorgenommen. Ackerman/Webelhuth beschreiben das Prädikat als bilaterales, aus Form- und Inhaltsseite bestehendes Sprachzeichen im Sinne de Saussures. Kategoriale Spezifikationen wie die Zugehörigkeit zu einer Wortart werden dabei der Formseite zugeschlagen; die Inhaltsseite umfasst neben den morphosyntaktischen Spezifikationen vor allem den „functional-semantic content“, d. h. das Konzept und die dazugehörigen semantischen Rollen. Um Beziehungen zwischen Konzepten einschließlich ihrer Rollen geht es auch in der vorliegenden Untersuchung.

Da die Ereigniskonzepte also aus dieser Sicht nicht an die Versprachlichung durch eine bestimmte Wortart gebunden sind, können Implikationsbeziehungen 
zwischen Konzepten grundsätzlich auch unabhängig von den Wortarten formuliert werden, in denen die Konzepte jeweils gefasst sind. Implikationsrelationen sind folglich insgesamt auf drei Ebenen formulierbar:

(i) Implikation zwischen Sätzen/Aussagen: Hans schleicht $\rightarrow$ Hans bewegt sich fort,

(ii) Implikation zwischen Wortbedeutungen: schleichen $\rightarrow$ sich fortbewegen („Lexical entailment“ Miller/Fellbaum 1992: 219; Cruse 1986: 14f.),

(iii) Implikation zwischen Konzepten: SCHLEICHEN $\rightarrow$ SICH FORTBEWEGEN (vgl. Cruse 2002: 543).

Die wortartenunabhängige konzeptuelle Herangehensweise in (iii) ist für die Beschreibung von Relationen innerhalb des FVG von besonderer Bedeutung, da diese typischerweise ein als Nomen lexikalisiertes Ereigniskonzept enthalten, die Fügung als Ganze aber einen verbalen Kopf besitzt und damit in ihrer Gesamtheit ein Ereigniskonzept kodiert. Auf der Ebene der Konzepte kann man somit das als Nomen lexikalisierte Ereigniskonzept mit dem durch die VP ausgedrückten Ereigniskonzept in Beziehung setzen (dazu weiter unten).

\subsubsection{Implikationsrelationen bei FVG und komplexen Prädikaten}

Um das Besondere des Bedeutungsaufbaus von FVG zu fassen, ist hier zunächst ein Blick auf den Bedeutungsaufbau komplexer Prädikate allgemein erforderlich. Als ,komplexe Prädikate‘ kann man mit Butt (2010) Konstruktionen bezeichnen, die zwei (oder mehr) prädikative Elemente enthalten, die zu einer prädikativen Einheit verbunden werden:

[...] the term complex predicate is used to designate a construction that involves two or more predicational elements (such as nouns, verbs, and adjectives) which predicate as a single unit, i.e. their arguments map onto a monoclausal syntactic structure (Butt 2010: 49). ${ }^{50}$

Der Definition Butts folgend kann eine ganze Reihe von Nomen-Verb-Verbindungen als komplexe Prädikate beschrieben werden. Dazu gehören sowohl freie Verbindungen von beliebigen Verben mit Ereignisnominalisierungen als auch Fügungen wie die in (1h)-(1i), die bisher vielfach als FVG beschrieben worden sind:

50 Der Begriff der „construction“ wird hier offensichtlich in einem sehr breiten Sinn und nicht als Terminus für ein konventionalisiertes Zeichen etwa im Verständnis Goldbergs (1995) verwendet. 
(1) a. Der Anwalt bereitet die Übernahme des Konzerns vor.

b. Der Junge beginnt ein Gespräch.

c. Der Vater überredet die Kinder zum Essen.

d. Der Vater zwingt die Kinder zum Lernen.

e. Der Staatsanwalt bringt den Zeugen zum Lachen.

f. Der Richter freut sich über die Aussage des Zeugen.

g. Sie freut sich über die Fahrt mit dem neuen Auto.

h. Der Lehrer zollt dem Kind Lob.

i. Das Mädchen hat Angst vor Wölfen.

Versucht man hier Relationen zwischen dem gesamten komplexen Prädikat und dem im Nomen ausgedrückten Prädikat zu formulieren, so ist die häufigste Relation wohl die einer Implikation des Nominalereignisses durch das Gesamtereignis: Wenn wie in (1c) DER VATER DIE KINDER ZUM ESSEN ÜBERREDET, dann kann aus der Gültigkeit des gesamten Ereignisses auch die Gültigkeit von ESSEN (KINDER) gefolgert werden. Zum Teil liegt aber auch eine spezielle Ausprägung der Implikationsrelation vor: In (1f) und (1g) impliziert das Gesamtereignis das Nominalereignis, von der Negation SICH NICHT FREUEN bzw. NICHT GENIESSEN kann aber ebenfalls auf die Gültigkeit von AUSSAGEN bzw. FAHREN geschlossen werden. Hier liegt somit ein Präsuppositionsverhältnis vor (man spricht hier auch von ,faktiven Verben‘, vgl. Levinson 1983: 181). Eine Implikation bzw. Präsupposition Gesamtereignis $\rightarrow$ Nominalereignis ist bei komplexen Prädikaten allerdings nicht zwingend gegeben, wie (1a) zeigt: Vom Gesamtereignis DIE ÜBERNAHME DES KONZERNS VORBEREITEN ausgehend kann nicht darauf geschlossen werden, dass das Nominalereignis DEN KONZERN ÜBERNEHMEN auch wirklich stattfindet (vorbereiten wäre dabei kein faktives Verb, sondern ein ,change of state verb‘, s. Levinson 1983: 181f.).

Für die hier besonders interessierenden Fälle (1h)-(1i) führt die Formulierung von Relationen zu einem überraschenden Ergebnis. Betrachtet man etwa (1h), so ist festzuhalten, dass das komplexe Prädikat und das Nomen dieselbe Bedeutung haben, d. h. dasselbe Konzept bezeichnen:

- Lob zollen bezeichnet ein Konzept LOBEN.

- Lob bezeichnet ein Konzept LOBEN.

Aufgrund dieser Übereinstimmung kann im Fall von Lob zollen sowohl von der Bedeutung der gesamten Fügung auf die Bedeutung des Nomens als auch umgekehrt von der Bedeutung des Nomens auf die Bedeutung der gesamten Verbin- 
dung geschlossen werden. Während die Implikation Gesamtbedeutung $\rightarrow$ Nominalbedeutung, wie die oben diskutierten Beispiele zeigen, keine Besonderheit von Fügungen wie Lob zollen darstellen (vgl. 1c oder 1g), ist die umgekehrte Folgerungsbeziehung Nominalbedeutung $\rightarrow$ Gesamtbedeutung bei komplexen Prädikaten sonst nicht anzutreffen (vgl. auch 2.7). Hier liegt also ein Alleinstellungsmerkmal vor, das sehr gut dazu geeignet ist, eine besondere Klasse von NomenVerb-Verbindungen zu begründen. Die Relation ,Nominalbedeutung impliziert Gesamtbedeutung der N+V-Verbindung' soll daher hier auch als Ansatzpunkt für die Neudefinition der Klasse FVG dienen. Diese Klasse wäre zwar am treffendsten als ,implikative Fügung، o. ä. zu etikettieren; da jedoch eine nicht unbeträchtliche Schnittmenge mit der Klasse der FVG in den hergebrachten Definitionsansätzen besteht, soll im Folgenden an dem einmal eingeführten Begriff festgehalten werden.

\subsubsection{FVG mit tautologischem Bedeutungsaufbau}

Die am Beispiel von Lob zollen beschriebene Implikationsrelation stellt, logisch gesehen, eine Tautologie dar, wie die Formulierung in (2) deutlich vor Augen führt:

(2) Wenn loben (x, y), das durch das Nomen ausgedrückte Ereigniskonzept der Fall ist, ist auch loben (x, y), das durch die gesamte Konstruktion ausgedrückte Ereigniskonzept der Fall.

Einen tautologischen Bedeutungsaufbau zeigen auch die Passivumschreibungen zur Aufführung/Versteigerung/Verteilung kommen, da hier ein Nomen in passivischer Bedeutung vorliegt. Dies ist freilich zu erläutern, da Nomina wie Aufführung/Versteigerung/Verteilung hinsichtlich ihrer Diathese grundsätzlich nicht festgelegt sind, sondern sowohl eine aktivische als auch eine passivische Lesart aufweisen können, vgl. die Beispiele in (3)-(5).

(3) a. die Aufführung des Stückes (= passivisch).

b. die Aufführung des Regisseurs (= aktivisch).

(4) a. die Versteigerung des Bildes (= passivisch)

b. die Versteigerung des Auktionators (= aktivisch)

(5) a. die Verteilung des Kuchens (= passivisch)

b. Verteilung des Vaters (war ungerecht) (= aktivisch) 
Beim Nomen im FVG zur Aufführung kommen wie in (6) ist indes keine aktivische Lesart möglich.

(6) Das Stück kommt zur Aufführung.

Dies ergibt sich schon aus der Tatsache, dass die Fügung zur Aufführung kommen eine Passivperiphrase ist. Dementsprechend kann dem Nomen Aufführung auch keine Agens-Rolle zugewiesen werden - andernfalls könnte das Nomen nicht als Träger der Passivbedeutung fungieren. ${ }^{51}$ Für Aufführung ist in diesem Fall somit die passivische Lesart AUFGEFÜHRT WERDEN (PATIENS) als alleinige Deutungsmöglichkeit anzusetzen. Aufführung kodiert damit dasselbe Konzept, das auch die gesamte Fügung zur Aufführung kommen ausdrückt.

Hierbei mag sich die Frage stellen, ob die behauptete Implikation Nominalbedeutung $\rightarrow$ Gesamtbedeutung auch in negierten Sätzen wie (7) gilt:

(7) Das Stück kommt nicht zur Aufführung.

Immerhin ließe sich argumentieren, dass Aufführung AUFGEFÜHRT WERDEN (STÜCK) die Bedeutung STÜCK WIRD NICHT AUFGEFÜHRT eindeutig nicht impliziert, dass somit ein negiertes nicht zur Aufführung kommen im Unterschied zu der entsprechenden affirmativen Fügung keinen implikativen Bedeutungsaufbau aufweist. Dies würde den hier gemachten Vorschlag, FVG nach der Implikation Nominalbedeutung $\rightarrow$ Gesamtbedeutung zu bestimmen, ad absurdum führen, da dann zur Aufführung kommen als FVG zu gelten hätte, nicht zur Aufführung kommen aber kein FVG wäre. Gesamtbedeutung und Satzbedeutung sind indes nicht gleichzusetzen: Unter Gesamtbedeutung wird hier, wie oben bereits angesprochen, lediglich die lexikalische Bedeutung des gesamten FVG - abzüglich grammatischer Merkmale wie Person, Numerus und Tempus - verstanden, nicht die Bedeutung des gesamten Satzes. Die Implikationsrelation Nominalbedeutung $\rightarrow$ Gesamtbedeutung wird damit durch eine Negation wie in (7) nicht aufgehoben, da die Negation auf der Satzebene bzw. auf der Ebene der Proposition operiert, nicht aber auf lexikalischer Ebene. Dass hier der Geltungsbereich der Negation die gesamte Proposition umfasst - man spricht in einem solchen Fall traditionellerweise von

51 In Sätzen wie (6) kann ein Agens lediglich durch eine durch-PP ausgedrückt werden: Das Stück kommt durch den Regisseur zur Aufführung; aktivisches Der Regisseur bringt das Stück zur Aufführung ist demgegenüber sicher die präferierte Option. 
„Satznegation“ -, zeigt die Umwandlung von (8a) in (8b) (zu diesem Test vgl. Duden-Grammatik 922):

a. Das Stück kommt nicht zur Aufführung.

b. Es ist nicht der Fall, dass das Stück zur Aufführung kommt.

Im Übrigen gilt nicht nur für FVG, sondern auch für einfache Verben, dass in einem Satz wie Hans lacht nicht sich gegenüber Hans lacht lediglich der propositionale Gehalt des Satzes, nicht aber der lexikalisch-konzeptuelle Gehalt von lacht ändert.

Ähnlich liegt der Fall bei der Negation mit dem quantifizierenden Determinierer kein, vgl. (9):
a. Er zollte ihr kein Lob.
b. Er gab keine Antwort.
c. Er stattete ihr keinen Besuch ab.

Kein ist zwar Kopf der NP (bzw. genauer: der DP), trotzdem liegt auch hier eine Negation der gesamten Proposition vor (,Satznegation“, vgl. auch Heidolph/Flämig/Motsch 1981: 220). Das zeigt die Umformung von (10a) in (10b):

(10) a. Er zollte ihr kein Lob.

b. Es ist nicht der Fall, dass er ihr Lob zollte.

Der lexikalisch-konzeptuelle Gehalt von Lob zollen bleibt durch die Satznegation somit unangetastet. Daher kann auch hier eine Implikationsrelation zwischen der Nominal- und der Gesamtbedeutung postuliert werden. Die Implikationsrelation gilt hier folglich für die Ebene des Lexikons und nicht für Sätze, in denen die Lexikoneinträge realisiert sind. ${ }^{52}$

52 Auch diese Unterscheidung kann übrigens sehr gut im Rahmen der von Ackerman/Webelhuth (1998) entwickelten Theorie des Prädikats formuliert werden. Diesem Ansatz zufolge ist die Inhaltsseite des als Zeichen aufgefassten Prädikats in zwei Schichten zu unterteilen, nämlich zum einen in den oben erwähnten „functional semantic content“, zum anderem in den „morphosyntactic content“, der neben den Tempus- und Aspektmerkmalen u. a. auch die Negation umfasst. Die hier relevante Implikationsrelation gilt somit nur für den ,functional-semantic content“ (einschließlich der semantischen Rollen), vgl. Ackerman/Webelhuth (1998: 6f.). 
Einen tautologischen Bedeutungsaufbau weisen neben den erwähnten Fügungen mit zollen und kommen prinzipiell auch Verbindungen mit geben, haben und machen auf, vgl. dazu die folgenden Beispiele mit den entsprechenden Testformulierungen:

(11) Antwort geben: Wenn AnTwORTEN (x), das durch das Nomen Antwort ausgedrückte Ereigniskonzept der Fall ist, ist auch ANTWORTEN (x), das durch die gesamte Konstruktion Antwort geben ausgedrückte Ereigniskonzept, der Fall.

(12) eine Reise machen: Wenn REISEN (x), das durch das Nomen eine Reise ausgedrückte Ereigniskonzept der Fall ist, ist auch REISEN (x), das durch die gesamte Konstruktion eine Reise machen ausgedrückte Ereigniskonzept, der Fall.

(13) Angst haben: Wenn sich FÜRCHTEN (VOR) (x, y), das durch das Nomen Angst (vor) ausgedrückte Ereigniskonzept der Fall ist, ist auch sich FÜRCHTEN (VOR) (x, y), das durch die gesamte Konstruktion Angst haben ausgedrückte Ereigniskonzept, der Fall.

Die These, dass in diesen Fällen von der Bedeutung des Nomens auf die Bedeutung des gesamten Gefüges geschlossen werden kann und daher ein implikativer Bedeutungsaufbau vorliegt, bedarf allerdings in einem wichtigen Punkt einer Präzisierung. Ein Konzept wie REISEN (x) ist im Hinblick auf seinen Aspekt grundsätzlich unterspezifiziert: Es kann sich um ein einmaliges REISE-Ereignis mit einem bestimmten Reiseziele handeln wie in dem Satz (14a), es kann aber auch z. B. ein zeitlich nicht determinierter Sachverhalt wie in (14b) vorliegen.

(14) a. Peter reist nach Südafrika.

b. Peter reist gerne.

Die N-Position in dem FVG eine Reise machen ist durch den unbestimmten Artikel allerdings quantifiziert. Entsprechend ist auch die Gesamtbedeutung von eine Reise machen quantifiziert und entspräche einem Accomplishment (in der Klassifikation der Ereignistypen von Vendler 1967 und Dowty 1979). Die Fügung kann daher nur z. B. für (14a) eintreten, nicht für (14b). Steht die DP im Plural (Reisen machen), so ist die Gesamtbedeutung, wie erwartbar, zeitlich nicht determiniert; sie entspricht damit einer Activity. An der Tatsache, dass auch hier von der Bedeutung des Nomens - genauer gesagt der NP - auf die Gesamtbedeutung des FVG geschlossen werden kann, ändert das nichts. Im Gegenteil, die semantische Abhängigkeit der gesamten Fügung von der NP wird dadurch noch deutlicher. 
Das Beispiel Angst haben in (13) unterstreicht noch einmal, dass eine etymologische Verwandtschaft zwischen dem Nomen und einem der Gesamtbedeutung entsprechenden einfachen Verb keine zentrale Rolle bei der Definition von FVG spielt (s. dazu auch Kapitel 2.4.1). Dass Angst haben am einfachsten durch sich fürchten zu ersetzen ist, während das etymologisch verwandte sich ängstigen stilistisch als markiert gelten muss, spielt für die Feststellung einer Implikationsrelation zwischen Nominal- und Gesamtbedeutung keine Rolle.

\subsubsection{FVG mit konversem Bedeutungsaufbau}

Die häufigen FVG mit bringen (s. dazu auch die Aufstellung in Kapitel 5.2) bilden hinsichtlich ihres Bedeutungsaufbaus eine besondere Gruppe. Das Nomen dieser Fügungen ist passivisch zu lesen (s. o. die Beispiele (3)-(5), vgl. auch Heringer 1968: 66; Seifert 2004: 76). Die Nomina in zur Aufführung/zum Abschluss/zur Anzeige bringen sind folglich als ABGESCHLOSSEN WERDEN, AUFGEFÜHRT WERDEN, ANGEZEIGT WERDEN usw. zu paraphrasieren. Vgl. dazu zunächst die folgenden Beispielsätze:

(15) a. Der Regisseur bringt das Stück zur Aufführung.

b. Der Student bringt seine Arbeit zum Abschluss.

c. Die Polizistin bringt das Verbrechen zur Anzeige.

Grundsätzlich können die entsprechenden Nomina, wie oben anhand der Beispiele (3)-(5) bereits dargelegt, sowohl aktivisch als auch passivisch interpretiert werden. Schreibt man dem Verbalabstraktum in (15a) versuchsweise aktivische Bedeutung zu, müsste Stück AGENS sein. Diese Interpretation ist jedoch wenig überzeugend, da dem Nomen Stück dann wesentliche Eigenschaften, die einen Agens ausmachen (bewusstes Involviertsein, Empfindungsfähigkeit usw., vgl. Dowty 1991: 275), fehlen. Auch für (15b) und (15c) wäre die Annahme absurd, Arbeit als Agens von Abschluss oder gar Verbrechen als Agens von Anzeige zu lesen. Wenn man dem Akkusativobjekt dagegen die Patiens-Rolle zuschreibt und die deverbalen Nomina Aufführung, Abschluss, Anzeige dementsprechend passivisch deutet wie z. B. in (16), ergibt sich eine wesentlich plausiblere Interpretation.

$$
\text { Der Regisseur (AGENS) bringt das Stück (PATIENS) zur Aufführung. }
$$

Der konzeptuelle Gehalt des Nomens Aufführung soll daher als passivisches AUFGEFÜHRT WERDEN (PATIENS, AGENS) notiert werden, wobei die Reihenfolge (PATIENS, 
AGENS) die für passive Ausdrücke kennzeichnende Aufmerksamkeitsfokussierung auf den PATIENS markiert (vgl. Langacker 1999: 49 sowie die traditionelle Unterscheidung zwischen aktivischer, d. h. agenszugewandter, und passivischer, d. h. agensabgewandter Perspektive bei Helbig/Buscha 2001: 146; vgl. auch Primus 2012: 12).

Die Besonderheit des Bedeutungsaufbaus der Fügungen des Typs zur Aufführung bringen gegenüber den im vorigen Abschnitt behandelten FVG besteht nun darin, dass das Nomen passivisch, das gesamte Gefüge jedoch aktivisch ist: zur Aufführung bringen bedeutet AUFFÜHREN (x, y). Zwischen AUFFÜHREN (x, y) als der Gesamtbedeutung und AUFGEFÜHRT WERDEN (y, $\mathrm{x}$ ) als der Bedeutung des Nomens besteht gleichwohl ein Implikationsverhältnis, wie die Formulierung in (17) zeigt:

(17) Wenn AUfGeFÜHRt WERdEn (PATIENS, AgENS), das durch Aufführung ausgedrückte Ereigniskonzept, der Fall ist, ist auch AUFFÜHREN (AGENS, PATIENS), das durch die gesamte Konstruktion versprachlichte Ereigniskonzept, der Fall.

Unterschiedliche Perspektivierungen ein- und desselben Sachverhalts - etwa das Gegenüber von jmd. verkauft jmdm. etwas und jmd. kauft etwas von jmdm. oder auch von Paul schlägt Hans vs. Hans wird von Paul geschlagen - werden in der Regel als Konversen beschrieben (vgl. Lyons 1971: 478-480; Lutzeier 1995: 85f.; Primus 2012: 12). Die Konverse ist ein besonderer Typ der Implikationsrelation. Hier gilt die wechselseitige Implikation trotz der jeweils unterschiedlichen Anordnung mindestens zweier Argumentstellen: Wenn KAUFEN (X, Y, z) der Fall ist, dann ist auch VERKAUFEN (Z, Y, X) der Fall.

Weil im Fall der FVG des Typs zur Aufführung bringen Nomen und gesamtes Gefüge zwei unterschiedliche Perspektiven auf ein identisches Ereignis ausdrücken, kann hier schwerlich von einem tautologischen Aufbau wie bei den in Abschnitt 3.2.3 behandelten Fügungen die Rede sein. Verbindungen dieses Typs sind stattdessen als FVG mit konversem Bedeutungsaufbau zu beschreiben.

\subsubsection{FVG mit meronymischem Bedeutungsaufbau}

\subsubsection{Imperfektive Verbindungen}

Die Frage nach dem Bestehen einer Folgerungsbeziehung zwischen Nominalund Gesamtbedeutung stellt sich auch für die N+V-Verbindungen des Typs im Aufbruch sein/im Bau sein, die zumindest bei Helbig/Buscha (2001: 71) auch als FVG klassifiziert werden; vgl. dazu zunächst die Beispiele in (18). 
(18) a. Die Gäste sind im Aufbruch/im Aufbruch begriffen.

b. Das Haus ist im Bau/im Bau befindlich.

c. Der Patient liegt im Sterben.

d. Der Wein ist noch in der Reifung.

Die Fügungen in (18) sind imperfektiv: Das Ereignis ist nicht abgeschlossen, und es ist als Teilphase eines umfassenderen Ereignisses markiert. Das umfassende Ereignis wird hier durch das Nomen ausgedrückt. Die Nomina bzw. substantivierten Infinitive sind hingegen als perfektiv zu bewerten, sofern man ihnen eine Ereignisstruktur zuschreiben kann. Dass es sich in der Tat um perfektive Ereignisnomina handelt, kann durch die Möglichkeit einer Kombination mit einem Attribut wie abgeschlossen/vollendet oder allmählich bzw. durch den syntaktischen Anschluss __ schreitet fort belegt werden. Das Attribut abgeschlossen/vollendet zielt auf die Frage, ob ein Ereignis einen Endpunkt besitzt; das Attribut allmählich und der Anschluss schreitet fort testet die inkrementelle Abarbeitung des Patiens, die für perfektive Ereignisse (d. h. für Accomplishments) typisch ist (s. Kapitel 5.2.5). Ereignisnomina mit imperfektiver Lesart lassen solche Attribuierungen jedenfalls nicht oder nur sehr bedingt zu, vgl. (19) gegenüber (20).

(19) a. der allmähliche Aufbruch, die Reifung/das Sterben/der Bau schreitet fort

b. der vollendete Bau, die vollendete Reifung

(20) a. ?der Kontakt schreitet fort, ?der Lauf schreitet fort, ?der Gang schreitet fort, ?das Sitzen schreitet fort

b. ?der allmähliche/'vollendete Kontakt, ?der allmähliche/?vollendete Lauf, ?das allmähliche/?vollendete Sitzen

Bei der Frage, ob ein Ereignisnomen als perfektiv oder imperfektiv zu gelten hat, spielt - wie bei Verben - selbstverständlich auch die syntaktische Umgebung eine wichtige Rolle: Der vollendete Lauf um den Harz ist akzeptabel, da dem Ereignis hier ein Endpunkt zugewiesen werden kann.

Wenn man auf das Verhältnis von Nominalbedeutung und Gesamtbedeutung der Fügung blickt, so ist jedenfalls festzustellen, dass die Nominalbedeutung perfektiv, die Gesamtbedeutung imperfektiv ist. Eine unmittelbare Folgerung von der Nominalbedeutung auf die Gesamtbedeutung ist also nicht möglich - eine Implikation wie in (21) ist nicht formulierbar.

?Wenn x STIRBT, ist x IM STERBEN BEGRIFFEN. 
Legt man für die Klasse FVG das oben erläuterte Implikationskriterium zugrunde, sind imperfektive $\mathrm{N}+\mathrm{V}$-Verbindungen mit perfektiven Ereignisnomina wie in (18) dann nicht als FVG zu klassifizieren.

Zwischen den Fügungen in (18) und FVG im hier definierten Sinne bestehen gleichwohl Übereinstimmungen, die nahelegen könnten, diese strikte Abgrenzung zu überdenken. Denn auch die Fügungen in (18) lassen einen Schluss von der Nominalbedeutung auf die Gesamtbedeutung zu, nämlich in Gestalt einer Teil-Ganzes-Relation. Inwiefern Teil-Ganzes-Relationen (Meronymien) Schlüsse vom Teil auf das Ganze bzw. umgekehrt zulassen, sei im Folgenden kurz erläutert.

Eine Teil-Ganzes-Relation zwischen Konzepten ist grundsätzlich insofern als Implikationsrelation beschreibbar, als im prototypischen Fall das Ganze stets seine Teile und ein Teil stets sein Ganzes impliziert (vgl. Miller/Fellbaum 1992: 220). Das bedeutet, auf Gegenstandskonzepte angewandt: Wenn FINGER gegeben ist, ist normalerweise auch HAND gegeben, und wenn HAND vorliegt, liegt im Regelfall auch FINGER vor. Die Implikationsrelation zwischen Teil und Ganzem ist jedoch etwas anders beschaffen als etwa eine Implikation zwischen einem Hyponym und seinem Hyperonym. Im zuletzt genannten Fall liegt die folgende Implikation vor:

(22) Wenn ein $\mathrm{x}$ eine HAND ist, ist $\mathrm{x}$ auch ein KÖRPERTEIL.

HAND ist also vollständig und unter allen Umständen als KÖRPERTEIL zu klassifizieren. Daher ist der klassische Testrahmen für lexikalische bzw. konzeptuelle Relationen, der Austausch des entsprechenden Wortes in einem ansonsten identischen Satz, uneingeschränkt anwendbar:

(23) Wenn die Aussage Werner brach sich die Hand der Fall ist, ist auch die Aussage Werner brach sich ein Körperteil der Fall.

Eine Implikation wie in (24) kann auch für Meronymien formuliert werden:

(24) Wenn die Aussage Werner legte die Hand auf den Tisch der Fall ist, ist auch die Aussage Werner legte die Finger auf den Tisch der Fall.

Diese Folgerungsbeziehung ist jedoch anders zu interpretieren als die Relation z. B. in (17), damit sie zu akzeptablen Sätzen führt. Zunächst muss stärker als bei der Implikationsbeziehung in (17) davon ausgegangen werden, dass (24) eine prototypische Situation beschreibt - es ist eben durchaus denkbar, dass unter 
Umständen die Hand auf dem Tisch liegt, die Finger aber nicht. Zu bedenken ist ferner, dass ein Ersatz von Hand durch sein Meronym Finger in zwei ansonsten identischen Sätzen zwar möglich ist, wie (24) zeigt, es aber auch Fälle gibt, in denen ein solcher Ersatz keine sinnvolle Folgerungsbeziehung ergibt, vgl. (25):

(25) Wenn die Aussage Werner brach sich die Hand der Fall ist, ist die Aussage Werner brach sich den Finger nicht (notwendigerweise) der Fall. ${ }^{53}$

Wird die Meronymie als Implikationsrelation beschrieben, ist dies somit nicht durch eine einfache Wenn-Dann-Formulierung überprüfbar, da ein Objekt X nicht zugleich sowohl Hand als auch Finger sein kann. Die Wenn-Dann-Formulierung, mit deren Hilfe Meronymie beschrieben werden kann, ist daher zu spezifizieren, etwa durch eine Formulierung wie ,innerhalb der Extension von X“.

(26) Wenn HAND der Fall ist, dann ist innerhalb der Extension von HAND auch FINGER der Fall.

Aufgrund der einschränkenden Formulierung ,innerhalb der Extension von X“ ist diese Implikationsrelation im Gegensatz $\mathrm{zu}$ der Implikation bei Hyperonymiebeziehungen aber gewissermaßen als ,nicht-kanonisch` $\mathrm{zu}$ bewerten.

Eine Teil-Ganzes-Relation zwischen dem Ereignis der N+V-Verbindung und dem im Nomen ausgedrückten Ereignis kann, wie oben angedeutet, auch für die Beispiele in (18) angesetzt werden: Hier stellt das in der gesamten Fügung kodierte Ereignis eine Teilphase des im Nomen kodierten Ereignisses dar. ${ }^{54}$ Die zeitlichen Relationen zwischen Ereignissen können daher wie in Abb. 1 dargestellt werden.

$53 \mathrm{Zu}$ diesem Beispiel vgl. auch die Diskussion bei Lutzeier (1995: 77).

54 Vgl. auch Starke (1989: 99), die zu den entsprechenden Verbindungen festhält, dass diese „weder den bloßen Prozeßverlauf noch das Resultat [...], sondern [...] einen zeitlichen Ausschnitt aus dem Prozeß “ wiedergäben. - Der Gedanke, dass Aspekt mit Hilfe von Teil-Ganzes-Relationen modellierbar ist, findet sich übrigens auch in der Aspekttheorie von Filip (2000: 80), die den Unterschied zwischen Perfektivität und Imperfektivität als Anwendung des Totalitätsoperators TOT bzw. des Partivitätsoperators PART auf abstrakte Prozessprädikate definiert. Perfektiva denotieren demzufolge nur ganze Ereignisse, während Imperfektiva Teilereignisse wiedergeben. 


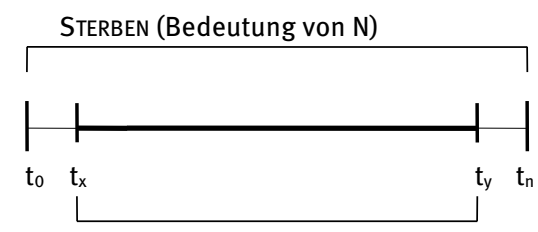

IM STERBEN SEIN (Bedeutung der N+V-Verbindung)

Abb. 1: Teil-Ganzes-Relationen bei im Sterben liegen

Eine Implikation Nominalbedeutung $\rightarrow$ Gesamtbedeutung kann also auch hier formuliert werden:

(27) Wenn sterben (Patiens), das durch das Nomen Sterben ausgedrückte Ereigniskonzept, in einem Zeitraum $\left(t_{0}-t_{n}\right)$ der Fall ist, ist für einen Zeitraum $\left(t_{x}-\right.$ $t_{y}$ ) auch eine Phase im Sterben sein (Patiens), das durch die gesamte Fügung ausgedrückte Konzept, der Fall; dabei gilt: $\mathrm{x}>0$ und $\mathrm{y}<\mathrm{n}$.

Wenn man also ein schwächeres Verständnis von Implikation, wie es in der Formulierung in (27) zum Ausdruck kommt, akzeptiert, können auch die imperfektiven Fügungen in (18) als FVG klassifiziert werden, freilich als nicht-prototypische Vertreter.

Die Annahme einer potentiell prototypischen Struktur für die Kategorie FVG steht hier allerdings auf einer anderen Grundlage als die in Kapitel 2 diskutierten Klassifikationsversuche, die angesichts der Schwierigkeit der Klassenabgrenzung ihr Heil in einer prototypischen Kategorienstruktur gesucht hatten. Das wesentliche Problem dieser Klassifikationen bestand darin, dass sie keine klaren Kriterien benennen konnten, welche eine Differenzierung zwischen zentralen und randständigen Kategorienvertreter möglich machen. Eine angebliche opinio communis, auf die sich etwa Seifert (2004: 54) und Eisenberg (2006a: 310) berufen, ist jedenfalls kein brauchbares Kriterium, und auch der ,sprachliche Mehrwert', der in der einen oder anderen Form immer wieder als Definitionskriterium genutzt wurde, ist nicht ausreichend objektivierbar und taugt daher nicht als Basis für eine prototypensemantische Hierarchisierung. Das Kriterium der Implikation Nominalbedeutung $\rightarrow$ Gesamtbedeutung hat dagegen zunächst den Vorteil, dass es durch Paraphrasetests überprüfbar ist. Die Nähe zum Prototyp der Kategorie korrespondiert schlicht mit der Möglichkeit, eine entsprechende Implikationsrelation zu formulieren, bzw. dem Grad des Gelingens einer entsprechenden Formulierung: Im Fall der tautologischen $\mathrm{N}+\mathrm{V}$-Verbindungen ist der Schluss von 
der Nominalbedeutung auf die Gesamtbedeutung am einfachsten, bei den Konstruktionen mit konversem Aufbau ist die Möglichkeit eines solchen Schlusses ebenfalls gegeben, allerdings unter der Voraussetzung eines Perspektivwechsels. Die zuletzt besprochenen imperfektiven Verbindungen lassen einen solchen Schluss ebenfalls zu, wobei hier eine Paraphrase aber nur unter verhältnismäßig starken Zusatzannahmen möglich ist.

Klarheit der Definition und eine prototypische Struktur der durch diese Definition konstituierten Kategorie schließen sich hier somit nicht aus. Dies entspricht im übrigen auch einer theoretischen Position innerhalb der Prototypensemantik, derzufolge Prototypikalität durchaus keine ,schwammigen“ Kategorien erzeugt, sondern, im Gegenteil, präzise Abgrenzungen erlaubt: „,...] we do not need the notion of fuzzy boundary: everything can be accounted for by variable construal of a normal, that is, determinate, boundary“, wie Croft/Cruse (2004: 95) festhalten.

\subsubsection{Inchoative Verbindungen (Typ ins Rollen kommen)}

Während bei Gegenstandskonzepten die zu einem Ganzen gehörigen Teile relativ leicht identifiziert werden können, ist dies bei Ereigniskonzepten schwieriger. Dementsprechend stellen Miller/Fellbaum (1992) zu Meronymien bei Verben fest:

[...] verbs cannot be taken apart in the same way as nouns, because the parts of verbs are not analogous to the parts of nouns. [...] The referents of verbs [...] do not have the kind of distinct parts that characterize objects, groups, or substances.

(Miller/Fellbaum 1992: 218)

Meronymien bei Verben könnten daher, so Miller/Fellbaum weiter, nicht absolut, sondern nur in Relationen bestimmt werden, genauer gesagt in ,the temporal relation between the activities that the two verbs denote“ (1992: 218). ${ }^{55}$ Auch wenn Meronymie bei Verben bzw. Ereigniskonzepten nur relativ bestimmbar ist, können gleichwohl auch hier gut definierte Teil-Ganzes-Relationen herausgearbeitet werden, wie die zeitlichen Verhältnisse bei imperfektiven $\mathrm{N}+\mathrm{V}$-Verbindungen gezeigt haben, s. o. Abb. 1.

55 Vgl. auch die Klassifikation unterschiedlicher Meronymie-Typen, die Winston/Chaffin/Herrmann (1987) aufgestellt haben. Meronymien bei Ereigniskonzepten werden hier grundsätzlich dem Typus „phase-process“ zugeordnet (1987: 426). 
Im Vergleich mit den Imperfektiva sind die einschlägigen Relationen bei inchoativen $\mathrm{N}+\mathrm{V}$-Verbindungen allerdings schwieriger zu erfassen. Dies sei am Beispiel der Fügung ins Rollen kommen demonstriert (hier in der konkret-räumlichen Lesart wie in Der Wagen kommt ins Rollen). Die Ereigniskonstellation innerhalb der Konstruktion kann man so beschreiben, dass der substantivierte Infinitiv Rollen ein Ereigniskonzept ROLLEN (x), das gesamte Gefüge ANFANGEN ZU ROLLEN (x) kodiert. Beide Konzepte stehen in einer festen Abfolge: ANFANGEN ZU ROLLEN stellt die Eintrittsphase zum voll entfalteten Ereignis ROLLEN dar. Die Schwierigkeit liegt darin, dass ANFANGEN ZU ROLLEN einerseits als eine Teilphase von ROLLEN verstanden werden kann, es andererseits aber auch kein ,guter ' Teil von RoLlen (x) ist, da unklar ist, inwieweit die Eintrittsphase bereits ganz zum ROLLEN-Ereignis gerechnet werden kann (vgl. Abb. 2; dazu allgemein auch die Überlegungen zur Entfaltung von Ereignissen in Ballmer/Brennenstuhl 1986: 57).

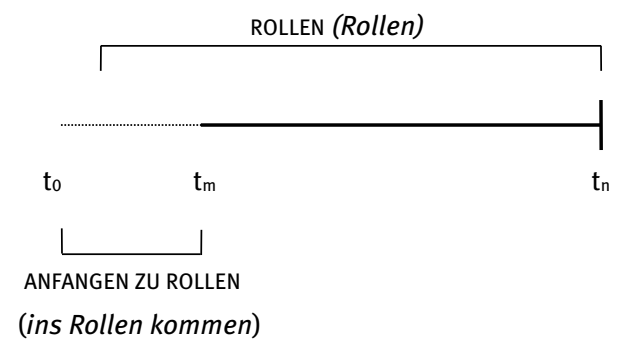

Abb. 2: Teil-Ganzes-Relationen bei ins Rollen kommen

Das Teil-Ganzes-Verhältnis zwischen ANFANGEN ZU ROLLEN und ROLLEN kann grundsätzlich auch als Implikation formuliert werden; allerdings ist dabei angesichts der oben geschilderten nicht ganz klaren Teil-Ganzes-Relationen eine starke Einschränkung zu formulieren:

(28) Wenn rollen ( $\mathrm{x})\left(\mathrm{t}_{0}-\mathrm{t}_{\mathrm{n}}\right)$, das durch Rollen ausgedrückte Ereigniskonzept, der Fall ist, ist innerhalb der zeitlichen Extension von Rollen auch ein Ereignis anfangen zu rollen (x) der Fall, zumindest zu einem Teil.

\subsubsection{Zwischenfazit: Implikation als gradierbares Konzept}

Fasst man, wie oben angedeutet, Implikation als gradierbares Konzept auf und nimmt man entsprechend eine Skala an, die von ,hundertprozentigen' bis hin zu 
lediglich eingeschränkt gültigen Schlüssen reicht, so wäre die in (28) formulierte Relation sicherlich als die schlechteste unter den hier bisher untersuchten Implikationsrelationen der Fügung zu beschreiben. Legt man die Implikation Nominalbedeutung $\rightarrow$ Gesamtbedeutung als Kriterium für den Ansatz einer Klasse von $\mathrm{N}+\mathrm{V}$-Verbindungen zugrunde, so kann man insgesamt eine Kategorienstruktur wie in Abb. 3 annehmen. In deren Zentrum stehen die tautologischen Fügungen (Angst haben, zur Aufführung kommen), während in einer weiteren zentrumsnahen Schicht die Verbindungen mit konversem Aufbau zu verorten sind (der Typus zur Aufführung bringen). Relativ weit vom Kategorienzentrum entfernt sind imperfektive Konstruktionen anzusiedeln, und die inchoativen Fügungen (ins Rollen kommen) schließlich bilden die Peripherie der Kategorie.

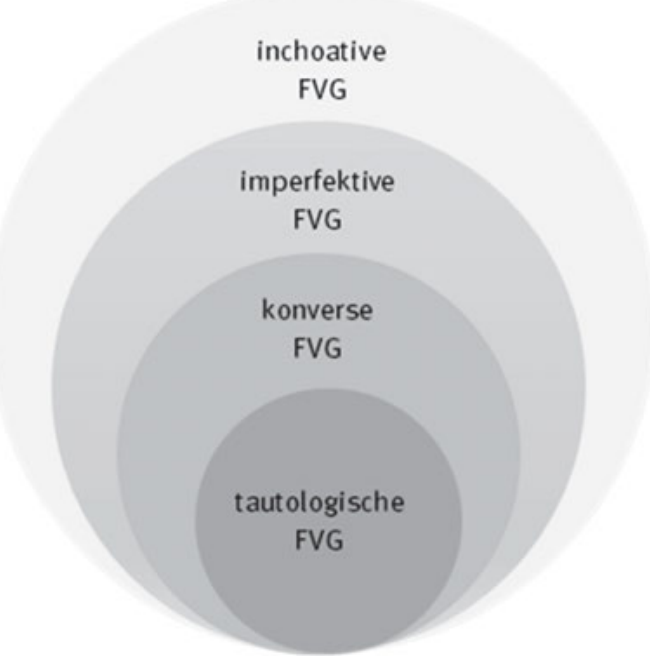

Abb. 3: Prototypische Struktur der Kategorie FVG

Eine solche Kategorie mit einem definierenden Kriterium, das in unterschiedlichen Graden oder Stufen vorliegt, stellt nun keine Ausnahme dar, sondern repräsentiert eine durchaus geläufige Kategorienstruktur. So sind nach Taylor (1995: 63f.) viele Kategorien über eine einzige essentielle Eigenschaft definiert, welche jedoch gewissermaßen unterschiedliche Reinheitsgrade aufweist: So kann (nach einem Beispiel Taylors) die Kategorie DREIECK klar als eine geometrische Figur bestimmt werden, die durch drei gerade und an den Enden aufeinanderstoßende Linien gebildet wird. Diese hinreichende und notwendige Eigenschaft, die die 
Grenze zwischen DREIECK und anderen geometrischen Figuren klar definiert, kann gleichwohl in unterschiedlichen Ausprägungen vorliegen: Ein von Hand gezeichnetes Dreieck, dessen Linien nicht ganz gerade sind und sich möglicherweise auch nicht berühren, ist ein schlechteres Exemplar der Kategorie als das Dreieck in einem Lehrbuch der Geometrie. Gleichwohl kann auch die von Hand gezeichnete Figur als Dreieck identifiziert werden und unter gewissen Umständen - z. B. als Illustration der Bedeutung von dt. Dreieck im Fremdsprachenunterricht - als hinreichend gutes Exemplar gelten.

\subsubsection{Weitere Eigenschaften von FVG: Typzuordnung und Kompositionalität}

Dass die unter der Kategorie FVG zusammengefassten Fügungen tatsächlich eine wichtige Gemeinsamkeit aufweisen, die diese von anderen $\mathrm{N}+\mathrm{V}$-Verbindungen unterscheidet, wird auch noch an einem anderen Punkt deutlich, nämlich der Typzuordnung der Fügungen. Wenn man versucht, N+V-Verbindungen jeweils einer übergeordneten konzeptuellen Kategorie, einem Typ zuzuordnen, so sind zwei verschiedene Möglichkeiten der Zuordnung identifizierbar. Bei N+V-Konstruktionen, die keine Implikationsrelation Nominalbedeutung $\rightarrow$ Gesamtbedeutung aufweisen und folglich nicht als FVG zu klassifizieren sind, erfolgt die Typzuordnung über das Verb als dem Kopf der Phrase. Dies gilt etwa für die meisten der eingangs in (1) erwähnten Beispiele, vgl. dazu nochmals (29) und (30).

(29) a. Der Vater zwingt die Kinder zum Lernen.

b. Der Staatsanwalt bringt den Zeugen zum Lachen.

(30) a. Der Richter freut sich über die Aussage des Zeugen.

b. Der Richter freut sich über die Aussage des Zeugen.

Die durch zum Lernen zwingen und zum Lachen bringen versprachlichten Ereignisse in (29) sind Untertypen eines BEWIRKEN-Ereignisses, und die Verbindungen in (30) sind vom Verb ausgehend als EMPFINDEN-Ereignisse zu typisieren. Diese Typzuordnungen können auch mit Folgerungsbeziehungen getestet werden, vgl. die Paraphrasen in (31).

(31) a. Wenn jemand seine Kinder zum Lernen zwingt, bewirkt er etwas.

b. Wenn jemand sich über etwas freut, empfindet er etwas. 
Bei FVG erfolgt die Typeinordnung nicht über das Verb als Kopf der Phrase, sondern über das Nomen: Zur Aufführung kommen bzw. bringen sind nicht von kommen bzw. bringen ausgehend als Bewegungs- oder Transportereignisse zu typisieren, sondern einer übergeordneten Kategorie ETWAS KÜNSTLERISCH DARBIETEN zuzuordnen, und Angst haben ist eindeutig ein psychisches Ereignis, kein BESITZEreignis; vgl. dazu die Paraphrasen in (32).

a. Wenn ein Stück zur Aufführung kommt, wird es künstlerisch dargeboten.

b. Wenn jemand Angst hat, dann fühlt sie oder er etwas..

Bei den peripheren FVG, d. h. den Imperfektiva und Inchoativa, ist die hierarchische Einordnung ebenfalls vom Nomen gesteuert, nicht vom Verb, vgl. die Folgerungsbeziehungen in (33), die eine Hierarchie ROLLEN - SICH BEWEGEN plausibel machen.

a. Wenn das Auto im Rollen ist, bewegt es sich.

b. Wenn das Auto ins Rollen kommt, bewegt es sich.

Das Kriterium der Typzuordnung unterstützt somit das oben eingeführte Implikationskriterium: Dort, wo eine gut formulierbare Implikation zwischen Nominalbedeutung und Gesamtbedeutung besteht, $\mathrm{d}$. h. bei tautologischen und konversen Verbindungen, erfolgt eine Typisierung über das Nomen. Imperfektive und inchoative Konstruktionen dagegen weisen neben einer schlechteren Formulierbarkeit der betreffenden Implikationsrelation auch eine Typzuordnung auf, mit der sie sich eher auf die Seite der regulären Nomen-Verb-Phrasen stellen, die ja stets über das Verb als ihren Kopf semantisch klassifiziert werden.

Die FVG-internen Differenzierungen, die sich hier im Hinblick auf die Implikationsrelation und die Typzuordnung feststellen ließen, korrespondieren ferner mit einem Unterschied bei der Kompositionalität der Verbindung: In jedem Fall als kompositional zu bewerten sind die Inchoativa und die Imperfektiva, und zwar insofern, als sich die inchoative bzw. imperfektive Gesamtbedeutung aus der Verbindung von Verb und Ereignisnomen ergibt. Die inchoative Bedeutung BEGINNEN ZU ROLLEN in (33b) kommt dadurch zustande, dass in etwas kommen als Ausdruck für BEGINNEN ZU mit einer nominalen Ereignisversprachlichung kombiniert wird. Für imperfektives in etwas sein und Rollen gilt dies analog.

Bei dem Versuch, den Bedeutungsaufbau tautologischer Fügungen wie Angst haben, Antwort geben oder zur Aufführung kommen zu beschreiben, ergeben sich indes Schwierigkeiten: Wenn, wie oben gezeigt, die Gesamtbedeutung 
der Fügung bereits aus der Bedeutung des Nomens vollständig erschließbar ist, stellt sich die Frage, welchen lexikalischen Bedeutungsbeitrag das Verb dann überhaupt noch in die Fügung einbringen kann. Dass ein Verb als Kopf der Phrase keinen semantischen Beitrag zur Bedeutung der VP leistet und dass das Verb damit semantisch leer ist, muss jedenfalls als ungewöhnlich gelten, da Verben im Regelfall eine Prädikation ausdrücken.

Bei konversem FVG (zur Aufführung bringen) verhält es sich etwas anders. Da das Nomen passivisch, die Gesamtfügung aber aktivisch ist, kommt dem Verb bringen hier immerhin die Funktion zu, die aktivische Perspektive auf den durch das Nomen ausgedrückten Sachverhalt zu markieren. Der Bedeutungsbeitrag des Verbs bleibt hier aber insofern überschaubar, als die Referenz des nominalen Ausdrucks in keiner Weise modifiziert wird - lediglich die Perspektive auf den Sachverhalt ist anders. Damit unterscheiden sich tautologische und konverse Verbindungen von den meronymischen in einem wichtigen Punkt: Bei den zuerst genannten FVG leistet das Verb keinen referenziellen Bedeutungsbeitrag; bei den FVG mit meronymischem Bedeutungsaufbau ist dies indes sehr wohl der Fall. Hier modifiziert das Verb auch die Referenz des gesamten Ausdrucks, da unterschiedliche Teilphasen des jeweiligen Ereignisses fokussiert werden. Dass Verben Referenz haben, dürfte der Normalfall sein; insofern weichen die tautologischen und konversen FVG am stärksten von diesem Normalfall ab und diese sind folglich auch am stärksten einer Erklärung bedürftig. Die Verbindungen mit meronymischem Bedeutungsaufbau stellen in dieser Hinsicht keine semantische Besonderheit dar. Dies berechtigt dazu, sie auch im weiteren Verlauf dieser Untersuchung nur am Rande zu behandeln.

\subsubsection{Die Semantik der Verben von FVG}

Auf der Basis der hier eingeführten Definition des Begriffs FVG ist auch eine erste Präzisierung der in der Forschung noch weitgehend impressionistisch beschriebenen Aufgabenverteilung zwischen Nomen und Verb eines FVG möglich. Wenn etwa Helbig (1984: 165) konstatiert, dass das Nomen in nicht näher definierter Weise die ,Hauptbedeutung، eines FVG trägt, so kann der Begriff ,Hauptbedeutung' hier präzisiert werden: Das Nomen trägt die Hauptbedeutung der Fügung, weil es die Gesamtbedeutung impliziert.

Vor diesem Hintergrund kann auch die Semantik der Verben von FVG näher bestimmt werden. Diese werden traditionellerweise als ,inhaltsarm', ,semantisch ausgebleicht‘ oder ähnlich bezeichnet (s. o. Kapitel 2.3.1). Beschreibungen dieser 
Art lassen im Allgemeinen jedoch kein Kriterium erkennen, nach dem ,Inhaltsarmut' bzw. deren Grad bestimmt werden kann. Auf der Basis der hier gewählten Klassenbestimmung lassen sich hingegen präzisere Angaben dazu machen, in welcher Hinsicht das Verb von FVG ,inhaltsarm' ist und wie verschiedene Grade von ,Inhaltsarmut' $z$ u unterscheiden sind:

- Bei den tautologischen Verbindungen hat das Verb keinen Anteil an der Sachverhaltsbeschreibung der Fügung; da die lexikalische Gesamtbedeutung der Fügung bereits vollständig aus der Nominalbedeutung her erschließbar ist, $d$. h. ohne dass das Verb eine lexikalisch-semantische Information in die Fügung einbringt, bedeutet ,inhaltsarm‘ hier schlicht ,ohne lexikalisch-semantische Merkmale‘.

- Bei FVG mit konversem Bedeutungsaufbau ist der Anteil des Verbs höher zu veranschlagen. Zwar beziehen sich in diesem Fall Nomen und gesamtes FVG ebenfalls auf denselben Sachverhalt, die agenszugewandte Perspektive, die ein aktivisches FVG zur Aufführung bringen gegenüber dem passivischen Nomen Aufführung (s. 2.4) ausdrückt, wird durch bringen wiedergegeben. ,Inhaltsarm' ist das betreffende Verb also insofern, als es keinen eigenen Sachverhalt kodiert, sondern lediglich den durch das Nomen vorgegeben Sachverhalt anders perspektiviert.

- Während bei tautologischen und konversen FVG das Verb keine eigene Sachverhaltsbeschreibung leistet, $d$. h. keine Referenz hat, ist bei den meronymischen Fügungen insofern ein eigener referentieller Beitrag des Verbs erkennbar, als dieses die jeweilige Teilphase des durch das Nomen angegebenen Ereignisses spezifiziert: Bei den Inchoativa ist dies die ,Anlaufphase‘ des Ereignisses, bei den Kursiva eine Teilphase im Ereignisverlauf. Insofern trägt das Verb hier durchaus eine Bedeutung. Da es aber letzten Endes lediglich das Ereignis modifiziert, das im Nomen ausgedrückt ist, und keine zusätzlichen, gegenüber dem Nomen gänzlich neuen Inhaltsmerkmale in die Fügung einbringt, ist es im Vergleich zu Verben wie laufen oder schreiben lexikalisch ,arm‘.

Ein Beleg dafür, dass FV der ersten beiden Gruppen keine lexikalische Bedeutung haben, liefert die Nominalisierung der FVG. So kann ein FVG wie zur Aufführung kommen (tautologisch) und zur Aufführung bringen (konvers) nur durch Aufführung selbst und nicht durch eine entsprechende Phrase nominalisiert werden. Dies zeigen die Beispiele in (34)-(35), in denen ein FVG in einem Folgesatz wieder aufgenommen wird. 
(34) a. Das Stück kam zur Aufführung. Die Aufführung war glänzend.

b. Das Stück kam zur Aufführung. *Das Zur-Aufführung-Kommen war glänzend.

(35) a. Er brachte das Stück zur Aufführung. Seine Aufführung war glänzend.

b. Er brachte das Stück zur Aufführung. *Sein Zur-Aufführung-Bringen war glänzend.

Hätte bringen bzw. kommen hier eine lexikalische Bedeutung, wären diese Verben auch in der Substantivierung sichtbar. Als meronymisches Verb kann kommen indes durchaus substantiviert werden, vgl. (36).

(36) Das Ins-Rollen-Kommen der Lawine muss verhindert werden.

Da inchoatives kommen, wie oben dargelegt, einen eigenen referentiellen Beitrag zur Gesamtbedeutung der Fügung leistet, entspricht dies ganz der Erwartung. Was kausatives bringen angeht, das traditionellerweise $\mathrm{zu}$ den FVG gerechnet worden ist, hier aber aus der Klasse ansgeschlossen wird (Näheres dazu s. $u$. in 3.3.1), zeigt sich auch am Substantivierungstest, dass es über eine lexikalische Bedeutung verfügt. So ist z. B. das Kausativum zum Lachen bringen ohne Probleme als Phrase substantivierbar:

(37) Sie lernen das Spielen, Beruhigen, Halten, Singen und das zum Lachen bringen des Babys. (https://perspektiven-fuer-das-leben.de/familie/kinder/detail/geschwisterkinder/; Zugriff 06.04.2021)

Dass bringen als kausatives Verb im Gegensatz zu bringen als FV Bedeutung trägt, zeigt sich möglicherweise auch daran, dass es gelegentlich auch als Input für morphologische Derivationen in Frage kommt. Dies ist z. B. bei zum Lachen bringen der Fall, s. das Beispiel (38):

(38) Diese Karte ist für alle Superhelden, Tränentröster, Zum-Lachen-Bringer, Quatschmacher, [...] und Zöpfeflechter. (https://kleineprints.de/products/design-postkarte-super-papi; Zugriff 06.04.2021)

Auch bei den Termini Inverkehrbringung bzw. Inverkehrbringer ist bringen Input einer Derivation, vgl. (39). 
(39) a. Wenn eine Ausnahmeregelung zur Inverkehrbringung [...] vorliegt, kann angebaut werden, ohne dass die entsprechende Landesregierung informiert wird. (die tageszeitung, 10.05.2004, S. 2; Zugriff 06.04.2021)

b. Weiters haben diese Inverkehrbringer den Verbraucher zu informieren über die Gefährdung, die von einem Produkt ausgeht. (Salzburger Nachrichten, 27.01.1995; Zugriff 06.04.2021)

Bei FV im hier definierten Sinne ist eine Ableitung von bringen nicht möglich. Die Aussagekraft von Bildungen wie Inverkehrbringer/Inverkehrbringung für die Frage nach dem Bedeutungsgehalt des Verbs ist jedoch nicht überzubewerten, da solche dezidiert fachlichen Prägungen durchaus Idiosynkrasien aufweisen können. ${ }^{56}$

\subsection{Abgrenzung gegenüber anderen Nomen-Verb-Syntagmen}

\subsubsection{Kausative (Typ zum Lachen bringen)}

Hält man die hier vorgestellte Klassenabgrenzung gegen die bisher üblichen Definitionsversuche von FVG, so sind, was den Bestand der Fügungen angeht, einige Unterschiede erkennbar. Die deutlichste Abweichung besteht wohl darin, dass bereits , wie angedeutet, Kausativkonstruktionen mit bringen und versetzen, die im allgemeinen Verständnis zum Kern der Klasse FVG gerechnet werden (vgl. van Pottelberge 2001: 301), ${ }^{57}$ nach den hier angewandten Kriterien nicht zur Klasse zählen; vgl. dazu die folgenden Beispiele:

(40) a. Der Verleger bringt den Autor zum Schreiben.

b. Der Clown bringt das Publikum zum Lachen.

c. Der kläffende Hund versetzt das Kind in Angst.

Der Grund dafür, dass der genannte Typ von Verbindungen hier nicht zu den FVG gerechnet wird, ist darin $\mathrm{zu}$ sehen, dass das geforderte Implikationsverhältnis

56 In dieser Hinsicht schwer zu beurteilen ist auch der juristische Terminus Tatbegehung, welcher sehr häufig auch durch einfaches Tat wiedergegeben werden kann.

57 Kritisch zur Zugehörigkeit von Kausativa haben sich Bahr (1977: 268) und Persson (1992: 156, 158) geäußert. 
nicht vorliegt, wie das Fehlschlagen der Testformulierung nach dem Muster von (17) zeigt (hier zu dem Beispiel 40a):

(41) *Wenn Schreiben (AgEns, PAtiens), das durch Schreiben ausgedrückte Ereignis, der Fall ist, ist auch schreiben Veranlassen (AgEns, ${ }_{1}$, AgEnS $_{2}$, PatiENS), das durch das gesamte FVG ausgedrückte Ereignis, der Fall.

Analog gilt auch für in Angst versetzen und zum Lachen bringen, dass mit dem Gegebensein eines Zustands SICH FÜRCHTEN (EXPERIENCER) bzw. eines Ereignisses LACHEN (AGENS) die jeweiligen bewirkenden Ereignisse nicht automatisch mitgegeben sind. Dies ist vor allem deshalb der Fall, weil das bewirkende Ereignis von einem prinzipiell beliebigen Agens ausgeführt werden kann: Von SICH FÜRCHTEN (KIND) ausgehend kann nicht geschlossen werden, dass der Träger des auslösenden Ereignisses Hund ist.

In diesem Zusammenhang ist allerdings darauf hinzuweisen, dass eine spezielle Subklasse von bringen-Konstruktionen, nämlich das sog. Pseudokausativum (von Polenz 1987: 184), durchaus den hier angelegten Kriterien entspricht und die dazugehörigen Fügungen daher als FVG zu gelten haben. Es handelt sich dabei um die bereits mehrfach erwähnten Konstruktionen des Typs zur Aufführung/zur Anwendung/zur Durchführung bringen. von Polenz (1987: 184) hat diese Verbindungen als „pseudokausativ“ bezeichnet, da der Agens des bewirkenden Ereignisses und der Agens des bewirkten Ereignisses referenzidentisch seien: Wenn eine Theatertruppe ein Stück zur Aufführung bringt, dann veranlasst diese nicht jemand Drittes, das Stück aufzuführen, sondern setzt die AUFFÜHREN-Handlung selbst ins Werk. Bei ,echten“ Kausativa, so von Polenz, seien die Agenten der betreffenden Ereignisse jedoch nicht referenzidentisch, wie auch das oben gegebene Beispiel (35) zeigt. Die bei den Pseudokausativa vorliegende Struktur ,Bewirken des eigenen Tuns‘ sei insgesamt - so von Polenz (1987: 184) in einer die sprachkritischen Einwände nachträglich bestätigenden Argumentation - als ,,semantisch künstlich und überflüssig“ zu bewerten und entsprechende NomenVerb-Verbindungen seien aus diesem Grund nicht als FVG zu betrachten.

Nach Maßgabe des Kriteriums ,Nominalbedeutung $\rightarrow$ Gesamtbedeutung“ sind diese Konstruktionen als FVG zu klassifizieren, vgl. dazu nochmals die Testformulierung in (42) zu dem Beispiel zur Aufführung bringen.

(42) Wenn AUfGEFÜHRT WERDEN (PATIENS, AgENS), das durch Aufführung ausgedrückte Ereigniskonzept, der Fall ist, ist auch AUFFÜHREN (AGENS, PATIENS), das durch die gesamte Konstruktion ausgedrückte Ereigniskonzept, der Fall. 
Wenn die sog. pseudokausativen bringen-Konstruktionen hier den FVG zugeschlagen werden, so befindet sich diese Entscheidung aber durchaus im Einklang mit einer Reihe von bisher vorliegenden Klassifikationsversuchen. So haben unter anderem auch Helbig (1984), Persson (1992), Seifert (2004) und Eisenberg (2006a) Fügungen dieses Typs als FVG betrachtet. Dabei werden unterschiedliche Begründungen für diese Entscheidung angeführt: Bei Helbig (1984: 169) spielt die Existenz von Kommutationsreihen mit den Verben kommen und z. T. auch sein eine wichtige Rolle, bei Eisenberg (2006a: 309f.) ist wohl die lokale bzw. direktionale Grundbedeutung des Verbs sowie die syntaktische Grundstruktur der Konstruktion ausschlaggebend für die Klasseneinordnung, Persson (1992: 155) und Seifert (2004: 78) berufen sich dagegen vor allem auf das Vorhandensein eines funktionalen Mehrwerts - hier in einer Betonung der „Nachhaltigkeit [...], mit der eine Handlung ausgeführt wird“ (Seifert 2004: 77). (Seifert [ebd.] bekennt sich dabei übrigens auch zu der Konsequenz, dass das Kriterium des Mehrwerts, den ein FVG gegenüber einem parallelen einfachen Verb aufweisen müsse, dazu führt, dass einzelne Pseudokausativa dann ggf. doch nicht zur Klasse zu rechnen sind; dies beträfe etwa in Anschlag bringen, zum Ausdruck bringen oder in Vorschlag bringen.)

Dass in der vorliegenden Untersuchung ,echte‘ kausative Konstruktionen mit bringen bzw. versetzen nicht berücksichtigt werden, ist leicht zu verschmerzen, da sich - soweit zu sehen - für Kausativa keine Beschreibungsvorteile ergeben, wenn man sie einer übergeordneten Klasse zuordnet. Im Übrigen muss es auch in wissenschaftshistorischer Hinsicht eigentlich als Kuriosum gelten, dass Kausativa durch die Beschreibung als FVG in gewisser Weise doppelt etikettiert worden sind. Wenn die Forschung sich als Reaktion auf die Sprachkritik von Beginn an bemüht hat, den systematischen Charakter kausativer Nomen-Verb-Verbindungen herauszustellen und diese damit zu ,rechtfertigen', so hat sie zwar eine zweifellos zutreffende Eigenschaft von Verbindungen mit bringen $z u+$ substantiviertem Infinitiv erfasst; sie hat aber damit gerade solche Konstruktionen zu salvieren gesucht, die nie einem Rechtfertigungsdruck gegenüber der Sprachkritik unterstanden. Die Kritik am Nominalstil hat sich in erster Linie an den sog. Pseudokausativa des Typs zur Aufführung bringen, zur Anwendung bringen entzündet, wie etwa die bekannten Auslassungen von Karl Kraus ${ }^{58} \mathrm{zu}$ diesem Thema illustrieren, während ,echte‘ Kausativa mit bringen oder versetzen kaum Stein des Anstoßes waren.

58 Karl Kraus hatte speziell die Fügung zur Abschaltung bringen kritisiert (vgl. Karl Kraus: Die Sprache. Hg. von Wolfgang Wagenknecht. Frankfurt a.M.: Suhrkamp 1987, 256 (Original in Die Fackel, Jg. 27, 1925, S. 99f.). 


\subsubsection{Phraseologismen mit PP/NP+Verb-Struktur (Typ jmdn. aus dem Häuschen bringen)}

$\mathrm{Zu}$ der Frage, ob feste Fügungen wie die in (43) zu den FVG zu rechnen sind, hat die Forschung bisher keine überzeugende und verbindliche Antwort gefunden.

aus dem Häuschen geraten/bringen/sein, über die Lippen kommen/ bringen, auf die Bühne kommen/bringen, auf die Palme bringen, in Harnisch sein/kommen/bringen

Teils werden diese Syntagmen strikt von den FVG abgegrenzt (Fleischer 1997: 137; van Pottelberge 2001: 234), teils werden sie grundsätzlich den FVG zugeschlagen (Klein 1968: 9; Rothkegel 1973: 54; Rostila 2011: 272), teils wird von Fall zu Fall entschieden, welche Konstruktion dazugehört oder nicht (Engelen 1968: 295; von Polenz 1987: 175f., 181-183; Seifert 2004: 63). von Polenz (1987: 175) begründet seine Entscheidung, eine Fügung wie auf die Palme bringen zu den FVG zu rechnen, eine Verbindung wie zu Papier bringen jedoch aus der Klasse auszuschließen, mit der jeweils unterschiedlichen Referenzfähigkeit der Nomina (ähnlich argumentiert bereits Klein 1968: 9). So beziehe sich das Nomen in auf die Palme bringen nicht auf einen konkreten Gegenstand, sondern sei mit ,in Wut' gleichzusetzen. Damit sei die Fügung einer Verbindung wie in Aufregung versetzen/geraten/sein vergleichbar, die von Polenz im Zentrum der Kategorie ansiedelt und bei der das Nomen einen Zustand, keinen konkreten Gegenstand bezeichne. Das Nomen in zu Papier bringen beziehe sich hingegen auf einen konkreten Gegenstand und gehöre damit nicht in die Klasse der FVG.

Nun bliebt freilich offen, wie die Referenzfähigkeit eines Nomens dingfest zu machen ist. von Polenz (1987: 175) selbst führt als Kriterium für Referenzlosigkeit die mangelnde Erfragbarkeit und Pronominalisierbarkeit des Nomens an (dazu auch Kapitel 6.6.5) und sieht auch in der „Reduzierung des Artikelgebrauchs“ ein Merkmal von FVG. Dass diese Kriterien ungeeignet sind, zeigt sich schon daran, dass auch bei zu Papier bringen - dessen Nomen übrigens wie das typischer FVG im Sinne von Polenz' ohne Artikel ist - sich unter diesen Tests nicht anders verhält als ein ,normales‘ FVG, vgl. (44).

(44) a. Er brachte sie zur Verzweiflung. - *Wozu brachte er sie?

b. Er brachte den Gedanken zu Papier. - *Wozu brachte er den Gedanken?

Gegner einer solchen Zuordnung von Phraseologismen wie in (43) zu den FVG bedienen sich eines ähnlichen Arguments, wie es von Polenz für den Ausschluss 
von zu Papier bringen vorgebracht hat. So weist Fleischer (1997: 137) darauf hin, dass die Nomina Häuschen, Lippen, Bühne keine nomina actionis darstellten und dass die betreffenden Fügungen daher von den FVG klassifikatorisch zu trennen seien. Ausnahmen wie von Polenz $(1987:$ 175, 183) sie für auf die Palme bringen, auf Touren bringen, aus dem Konzept bringen gelten lässt, nimmt Fleischer dagegen offenbar nicht an. Abgesehen davon, dass auch Fleischer kein Testverfahren anführen kann, mit dessen Hilfe angegeben werden kann, ob z. B. Konzept in aus dem Konzept bringen ein nomen actionis ist oder nicht, muss grundsätzlich eingewandt werden, dass Fleischers Beharren auf einem nomen actionis als notwendigem Bestandteil der Fügung willkürlich erscheint. Eine Begründung, weshalb ein nomen actionis notwendiger Bestandteil eines FVG sei, wird jedenfalls nicht vorgelegt. Eine solche Begründung wäre aber erforderlich, wenn die Klasse FVG nicht bloß eine Setzung sein soll, in der einzelne willkürlich ausgewählte Elemente miteinander kombiniert sind.

Das Einordnungsproblem, das sich für idiomatische Nomen-Verb-Verbindungen stellt, ist letztlich eine Folge der unzureichenden Definitionen, mit deren Hilfe die Klasse FVG bisher bestimmt worden ist. Legt man der Klassenbestimmung jedoch das Kriterium der Implikation Nominalbedeutung $\rightarrow$ Gesamtbedeutung zugrunde, ergeben sich klare Trennlinien: Eine solche Implikation kann bei Phraseologismen wie den oben genannten nicht formuliert werden, wie (45) deutlich macht.

(45) a. *Wenn BÜHNE der Fall ist, ist auch AUFGEFÜHRT WERDEN der Fall.

b. `Wenn LIPPEN der Fall ist, ist auch AUSGESPROCHEN WERDEN der Fall.

Auch bei nicht-präpositionalen Nomen-Verb-Verbindungen mit einem Konkretum (z. B. Gummi/Gas geben, sich die Kugel geben o.ä.), die zumindest aufgrund des Verbs eine vordergründige Übereinstimmung mit einem FVG wie Antwort geben aufweisen, liegt keine einschlägige Implikationsrelation vor:

(46) *Wenn GAS/Gummi der Fall ist, ist auch BESCHLEUnigen der Fall.

In den Fällen in (45) und (46) kann zwar nicht von den Nominalbedeutungen auf die Gesamtbedeutung geschlossen werden, es besteht aber immerhin ein lockerer inhaltlicher Zusammenhang zwischen Nomen und Gesamtbedeutung: In (45a) liegt zwischen BÜHNE und AUFGEFÜHRT WERDEN eine Kontiguitätsrelation 
HANDLUNG - TYPISCHER HANDLUNGSORT, ${ }^{59}$ bei LIPPEN und AUSGESPROCHEN WERDEN in (45b) eine Kontiguitätsrelation HANDLUNG - HANDLUNGSINSTRUMENT vor; bei GAS/GUMMI kann gleichfalls eine (allerdings sicher schwerer herzustellende) Kontiguitätsrelation zwischen Nominal- und Gesamtbedeutung angenommen werden. Diese könnte man als HANDLUNG - HANDLUNGSAUSLÖSENDER FAKTOR bzw. HANDLUNG - BEGLEITFAKTOR beschreiben. Durch das Bestehen dieser Kontiguitätsrelationen ist es immerhin ansatzweise möglich, zwischen Nominal- und Gesamtbedeutung einen inhaltlichen Zusammenhang zu konstruieren. Die Fügungsbedeutung ist hier somit zumindest teilweise vom Nomen her verständlich. Da das Nomen in diesen Fällen aufgrund der vorhandenen Kontiguitätsrelation eine Art Hinweis auf die Fügungsbedeutung enthält, könnte man - im Unterschied zu den durch ihren implikativen Bedeutungsaufbau gekennzeichneten FVG - von einem ,indizierenden Bedeutungsaufbau'sprechen. Letzten Endes sind die betreffenden Idiome aber nicht zu den FVG zu zählen, da deren definierendes Merkmal nicht vorliegt.

\subsubsection{Syntagmen mit Substantiv im Nominativ (Typ Die Zahlung erfolgt)}

Im Zusammenhang FVG sind gelegentlich auch Fügungen mit Verben wie erfolgen, geschehen, stattfinden, sich ereignen, passieren, eintreten u. ä. diskutiert worden, deren Subjektposition durch eine Ereignisnominalisierung eingenommen wird, vgl. (47).

(47) a. Die Zahlung der Restschuld erfolgt umgehend.

b. Die Prüfung findet um neun Uhr statt.

c. Der Verlust des Besitzes tritt nach der Ausreise ein.

Eine Gemeinsamkeit mit den FVG wird darin gesehen, dass auch hier der Kern der Satzaussage im Nomen enthalten ist, während das Verb keine eigenständige Bedeutung enthält (vgl. Helbig 1984: 167f.; von Polenz 1987: 170; Seifert 2004: 55). Der Unterschied zu den üblicherweise als FVG bezeichneten Fügungen besteht in der Distribution des Nomens: Während bei typischen FVG das Nomen stets Teil des Prädikatskomplexes ist, tritt das Nomen der hier in Rede stehenden Verbindungen als Subjekt auf. Aufgrund dieser abweichenden syntaktischen Eigenschaft werden Syntagmen wie in (47) bei Helbig (1984: 168), von Polenz (1987:

59 Zum Begriff Kontiguität vgl. Raible (1981/2011: 24f.). 
180) und Seifert (2004: 69f.) lediglich zur Peripherie der FVG gerechnet. ${ }^{60}$ Eine Begründung dafür, weshalb im Zentrum der Kategorie ,FVG' die Nomina Bestandteile des Prädikatskomplexes zu sein haben, liefern die Autoren nicht. Maßgeblich für die Entscheidung, was zentral ist und was zur Peripherie gehört, ist hier offenbar wiederum die Forschungstradition, die sich eben überwiegend nur für Prädikatskomplexe und weniger für Fügungen mit nominativischen Ereignisnominalisierungen interessiert hat. ${ }^{61}$

Befragt man die Syntagmen in (47) nach ihrem Bedeutungsaufbau, so ist zunächst vorstellbar, dass durchaus eine Implikationsrelation postuliert werden kann, vgl. die hier die folgende Formulierung zum Beispiel (47a):

(48) Wenn GEZAHLT WERDEN (PATIENS), das durch das Nomen ausgedrückte Ereigniskonzept, der Fall ist, ist gleichzeitig GEZAHLT WERDEN (PATIENS), das durch die gesamte Verbindung ausgedrückte Ereigniskonzept, der Fall.

Gleichwohl sind Fügungen dieses Typs nicht als FVG im hier definierten Sinne zu betrachten. Ein Grund dafür ist, dass ein Satz wie Die Zahlung erfolgt oder Das Fest findet statt Informationen pragmatischer Natur enthält: Hier wird der Satz vor dem Hintergrund der Annahme geäußert, dass das betreffende Ereignis auch nicht der Fall sein könnte. Eine solche pragmatische Information kann mit einem bloßen Nomen wie Zahlung oder Fest jedoch nicht verbunden sein. Ein vollständiger Schluss von der Nominalbedeutung auf die Gesamtbedeutung der Fügung ist daher nicht möglich.

Ein zweiter Grund, weshalb Sätze mit erfolgen, stattfinden usw. hier nicht weiter berücksichtigt werden, liegt in dem Status der Nomen-Verb-Verbindungen, d. h. schlicht in der Tatsache, dass hier Sätze vorliegen. Bisher sind hier lediglich VPs behandelt worden. Für diese lassen sich Lexikoneinträge formulieren, für Sätze hingegen - solange es sich nicht um Sprichwörter handelt - ist die Annahme eines eigenen Lexikoneintrags nicht angebracht. Die Klasse der FVG wäre $\mathrm{zu}$ heterogen, wollte man neben Lexikoneinträgen noch Einheiten der freien Syntax integrieren.

60 von Polenz (1987: 170) spricht zwar in Bezug auf Verben wie erfolgen, stattfinden usw. von „Nominalisierungsverben“; „Nominalisierungsverbgefüge“ bildeten diese Verben jedoch nicht, so von Polenz ohne weitere Begründung.

61 Dass sich in der Forschung zu FVG keine eingehendere Auseinandersetzung mit diesen Konstruktionen findet, wird auch von Cantarini (2004: 133) kritisch angemerkt. 


\subsubsection{Die Verlaufsform (Typ am/beim Essen sein)}

Die ältere germanistische Forschung, die sich überwiegend für die geschriebene Standardsprache interessiert hat, hat der sog. Verlaufsform oder Progressivkonstruktion (ich bin am Überlegen, ich bin beim Aufräumen) kaum Beachtung geschenkt. Erst mit der verstärkten Hinwendung der jüngeren Sprachwissenschaft auch zur gesprochenen Alltagssprache sind Phänomene wie die Verlaufsform in den Blickpunkt gerückt (vgl. Zifonun/Hoffmann/Strecker 1997: 1877-1880). Vor diesem Hintergrund verwundert es nicht, dass das Verhältnis von FVG und Verlaufsform - mit Ausnahme der wenig rezipierten Untersuchungen von Hinderdael (1981) und Klimaszewska (1983) - erst in den letzten Jahren zum Thema gemacht worden ist. ${ }^{62}$ So hat von Polenz, der die Verlaufsform in seinen maßgeblichen Beiträgen zur FVG-Problematik noch unberücksichtigt gelassen hat, in späteren Arbeiten auf die enge Verwandtschaft der FVG mit der Verlaufsform hingewiesen (1999: 352). Auch in der Grammatik von Helbig/Buscha (2001: 80) wird die Verlaufsform zu den FVG gerechnet, während der Beitrag von Helbig (1984) noch nicht auf diese eingeht. Der Zusammenhang von FVG und Verlaufsform wird ferner bei van Pottelberge (2001: 411-432) eingehend diskutiert.

Als Gemeinsamkeiten von FVG und Verlaufsform werden in der Forschungsliteratur verschiedene Eigenschaften genannt: Der vergleichbare formale Aufbau Präposition + Verbalnomen + inhaltsarmes Verb (van Pottelberge 2001: 411), die Kodierung einer Aktionsart - Hinderdael (1981: 351) zählt die Verlaufsform zu den kursiven FVG -, das Zutreffen wesentlicher syntaktischer Beschränkungen, die normalerweise als typisch für FVG gelten, so z. B. die fehlende Erfragbarkeit, Pronominalisierbarkeit und Attribuierbarkeit der Verlaufsform (Er ist am Arbeiten - ${ }^{*}$ Woran ist er?; *das Arbeiten, woran er ist; ${ }^{\star}$ er ist am unermüdlichen Arbeiten, vgl. van Pottelberge 2001: 411; zu diesen Kriterien s. Kapitel 2.4.2). Ferner ließe sich noch die Ersetzbarkeit der gesamten Konstruktion durch ein etymologisch verwandtes einfaches Verb (am/beim Essen sein - essen) anführen. Einen wesentlichen Unterschied zwischen FVG und Verlaufsform sieht van Pottelberge (2001: 422) jedoch darin, dass die Verlaufsform ein grammatisches Verfahren darstellten, während die FVG der Phraseologie zuzuordnen seien. Verlaufsformen könnten, so die Argumentation van Pottelberges, zu jedem intransitiven Verb des Deutschen gebildet werden, da jedes Verb über einen substantivierten Infinitiv

62 Nur sehr vereinzelt ist schon früher auf die Verwandtschaft von FVG mit der Verlaufsform eingegangen worden, vgl. Eichler/Bünting (1976: 91). Hier wird die Verlaufsform als Beispiel für eine „Streckung“ mit einem Funktionsverb genannt (Hinweis bei van Pottelberge 2001: 414). 
verfüge. Kursive FVG dagegen seien nicht zu jedem Verb bildbar. Deren Bildbarkeit setze vielmehr das Vorhandensein eines entsprechenden Nomens voraus. Zudem spiele die Idiomatik eine besondere Rolle, wie sich z. B. an den je eigenen Präpositionen bei in Furcht sein und auf der Flucht sein zeige. Dies spricht in der Tat dafür, kursive FVG wie die zuletzt genannten als Einheiten des Lexikons zu behandeln und sie vom grammatischen Verfahren der Verlaufsform zu trennen.

Im Hinblick auf den Bedeutungsaufbau bleibt aber festzuhalten, dass Verlaufsformen wie am Weggehen sein oder am Bauen sein sich von den oben als FVG behandelten Konstruktionen im Weggehen sein und im Bau sein nicht unterscheiden: ${ }^{63}$ In beiden Fällen wird ein intermediäres Stadium zwischen dem Beginn und Abschluss des jeweiligen Ereignisses bezeichnet. ${ }^{64}$ Die Verlaufsform könnte von diesem Standpunkt aus somit ohne Probleme als implikative Fügung und damit als FVG klassifiziert werden. Wie strikt die Unterschiede zwischen Grammatik und Lexikon zu handhaben sind, auf die van Pottelberge hinweist, und welche Bedeutung diesen Unterschieden für die sprachwissenschaftliche Kategorienbildung zugemessen wird, ist letztlich eine Frage der Sprachtheorie. In sprachtheoretischen Modellen, die ein Kontinuum zwischen Lexikon und Grammatik ansetzen, hätten diese Unterschiede weniger Gewicht als in Modellen, die beide Bereiche strikt trennen (eine weitergehende Diskussion dazu findet sich in Kapitel 4.3).

Auch wenn die Verlaufsform in einen Zusammenhang mit den FVG gestellt werden können, bleibt doch festzuhalten, dass diese dann allenfalls den meronymischen Fügungen und damit dem Rand der Kategorie FVG zuzurechnen wäre. Da Verbindungen mit meronymischem Bedeutungsaufbau in dieser Untersuchung ohnehin nicht im Mittelpunkt stehen, sei auch auf die Verlaufsformen nicht weiter eingegangen.

\subsubsection{Nicht-Transformativa (Typ in Bewegung bleiben)}

Zu den „Durativ-FVG“ rechnet von Polenz (1987: 174) nicht nur Fügungen wie in Bewegung sein, im Streit liegen, sondern explizit auch Verbindungen mit bleiben wie etwa in Bewegung, in Verbindung bleiben (vgl. auch Engelen 1968: 294; Tao

63 Explizit auf die übereinstimmende Semantik zwischen der Verlaufsform und Konstruktionen wie im Bau sein/befindlich sein $\mathrm{u}$. ä. weist auch Admoni (1982: 176) hin.

64 Vgl. Allerton (2001: 206) zum englischen Progressiv: Dieses beschreibe „a process [...] that is in progress and that we see neither the beginning nor the end of; in other words, we have only a partial view of this process“. 
1997: 76; Fabricius-Hansen 2006: 268). ${ }^{65}$ Auch Seifert (2004: 86) behandelt bleiben als FVG, spricht hier jedoch im Anschluss an Pape-Müller (1980: 162f.) und Schwall (1991: 192) statt von einer durativen Aktionsart von einer kontinuativen Aktionsart, um dem Unterschied zu den Fügungen des Typs in Bewegung sein gerecht $\mathrm{zu}$ werden. In zahlreichen anderen Untersuchungen, etwa von Bahr (1977), Helbig (1984) und Starke (1989), findet bleiben jedoch keine Erwähnung als FV. Der Status von bleiben als FV ist somit insgesamt unklar.

Setzt man, wie hier vorgeschlagen, eine Implikation Nominalbedeutung $\rightarrow$ Gesamtbedeutung als klassenkonstituierendes Kriterium an, kann der Stellenwert der Verbindungen mit bleiben eindeutig beurteilt werden. Bleiben ist als Verb - genauer: als Kopulaverb (Eisenberg 2006a: 85) - anzusehen, das intransformative Aktionsart zum Ausdruck bringt (Zifonun/Hoffmann/Strecker 1997: 1867). Intransformativa zeichnen sich dadurch aus, dass sie eine Aussage über ein Vorereignis enthalten, nämlich dass das Vorereignis mit dem durch das Verb selbst bezeichneten Ereignis identisch ist. Mit Kursiva wie in Bewegung sein usw. ist hingegen keine Aussage über ein Vorereignis verbunden, vgl. (49a) und (49b).

(49) a. X bleibt in Bewegung: Vorereignis = SICH BEWEGEN (PATIENS)

b. $\mathrm{X}$ ist in Bewegung: VOREREIGNIS $=$ ?

Das Nomen Bewegung enthält keinerlei Information über ein Vorereignis. Deshalb kann vom Nomen auch nicht auf die Gesamtbedeutung der Fügung geschlossen werden. Aus diesem Grund werden Nomen-Verb-Verbindungen hier nicht als FVG behandelt.

\subsubsection{Figura etymologica (Typ ein luxuriöses Leben leben)}

Eine offensichtliche Verwandtschaft mit FVG, auf die schon Bahr (1977: 79) hingewiesen hat, zeigt auch der herkömmlicherweise als figura etymologica bezeichnete Konstruktionstyp in (50), dessen Nomen ein Verbalabstraktum des jeweiligen Verbs darstellt.

(50) a. Ich lebe ein luxuriöses Leben.

b. Er geht einen schweren Gang.

c. Ein Christ tut gute Taten.

65 Zur problematischen Klasse der durativen bzw. kursiven FVG s. auch Kapitel 2.2.1. 
Ähnlich wie bei den tautologischen Fügungen des Typs Lob zollen oder Angst haben impliziert die Bedeutung der NP luxuriöses Leben die Gesamtbedeutung, die einfach mit ,luxuriös leben' angegeben werden kann. Vom Bedeutungsaufbau her stimmen figura-etymologica-Verbindungen mit den FVG, wie sie hier definiert sind, somit grundsätzlich überein.

Gleichwohl weist der Bedeutungsaufbau einer figura etymologica im Vergleich zu dem eines FVG weitere Eigenschaften auf. So ist zunächst festzuhalten, dass hier nicht nur von der NP auf die Gesamtbedeutung, sondern auch von der Gesamtbedeutung auf die Bedeutung der NP geschlossen werden kann. Bei FVG ist ein Schluss in beide Richtungen nicht möglich, vgl. (51) gegenüber (52).

(51) [[ein luxuriöses Leben] leben] ,luxuriös leben`

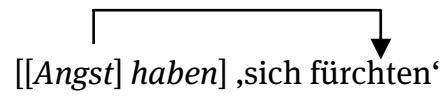

Ein Indiz dafür, dass figura-etymologica-Konstruktionen und FVG trotz der gegebenen Gemeinsamkeiten als je eigenständige Konstruktionstypen zu behandeln sind, bieten auch die Funktionen der beiden Konstruktionen. Soweit sich hierzu ohne eine umfassende Vergleichsuntersuchung überhaupt etwas sagen lässt, kann festgehalten werden, dass die figura-etymologica-Konstruktion vor allem dann verwendet wird, wenn eine Handlung oder ein Zustand modal bestimmt werden soll. Eine figura etymologica ohne Attribuierung ist daher fragwürdig bzw. nur in besonderen Kontexten akzeptabel, vgl. (53b).

(53) a. Er lebt ein luxuriöses Leben.

b. 'Er lebt ein Leben.

Auch eine idiomatisierte Fügung mit Possessivum wie die in (54) bestätigt diese Beobachtung, da hier ebenfalls die Lebensführung näher bestimmt wird.

(54) Er lebt sein Leben. 
Bei FVG ist die modale Bestimmung durch ein Attribut nicht obligatorisch (bis auf die isolierten Fälle eine gute/schlechte [...] Entwicklung nehmen und einen guten/schlechten [...] Verlauf nehmen, s. Helbig 1984: 174); vgl. dazu (55).

(55) a. Er hat Angst.

b. Sie macht einen Sprung.

c. Sie gibt Antwort.

Da es zudem zahlreiche FVG gibt, deren nominale Komponente grundsätzlich nicht attribuiert werden kann, vgl. (56), kann die zentrale Funktion von FVG sicher nicht in der modalen Bestimmung des jeweiligen Ereignisses gesehen werden (zur Attribuierung von FVG s. Kapitel 6.4; zur Frage der Funktionen s. Kapitel 7).

(56) ${ }^{\star}$ Er bringt das Stück zur gelungenen Aufführung.

\subsubsection{Syntagmen des Typs außer Gebrauch kommen/sein}

Fügungen wie in (58), deren PP durch die Präposition außer eingeleitet wird, sehen implikativen Fügungen wie ins Rollen kommen usw. auf den ersten Blick sehr ähnlich.

(57) a. außer Gebrauch sein/kommen/nehmen/setzen

b. außer Betrieb sein/nehmen/setzen

c. außer Dienst stellen

d. außer Kraft sein/treten

e. außer Gefecht sein/setzen

Ihr Bedeutungsaufbau scheint jedoch grundsätzlich anders beschaffen zu sein: Zwischen der Bedeutung des Nomens Gebrauch in (58a) und der Gesamtbedeutung (ALLMÄHLICH) NICHT MEHR GEBRAUCHT WERDEN besteht keine Implikationsrelation. Man könnte überlegen, ob dann nicht zumindest die PP außer Gebrauch die Gesamtbedeutung impliziert. Dies stünde jedoch im Gegensatz zum bisher praktizierten Verfahren, das stets nur von der Bedeutung des Nomens, nicht von der Bedeutung der Phrase ausging, in der das entsprechende Nomen vorkam. Außerdem ist unklar, welche Bedeutung der Phrase außer Gebrauch in einem solchen 
Fall zugesprochen werden müsste. Die Präposition außer kommt im Wesentlichen nur in phraseologischen Verbindungen vor (außer Haus, außer Atem usw.), daher kann ihr hier nur schwer eine wendungsexterne Bedeutung zuerkannt werden. Überhaupt ist mit den in (58) genannten Verbindungen wohl bereits der Hauptbestand der betreffenden Bildungen erschöpft, weshalb hier von einer isolierten Gruppe von idiomatischen Verbindungen auszugehen ist. So hat bereits Engelen (1968: 298) festgestellt: „Die Reihe mit der Präposition außer [...] ist sehr unproduktiv und weist fast nur lexikalisierte Beispiele auf“. Daher ist anzunehmen, dass es sich bei diesen wenigen Verbindungen um Analogiebildungen zu „echten“ FVG handelt.

\subsection{Fazit}

In den vorangegangenen Abschnitten wurde der Versuch unternommen, die bisher als FVG bezeichneten Nomen-Verb-Verbindungen nicht mehr auf der Basis eines angeblich gemeinsamen Sets von Funktionen, sondern nach ihrem Bedeutungsaufbau zu bestimmen. Als gut überprüfbares Kriterium, das eine Abgrenzung gegenüber anderen Nomen-Verb-Verbindungen ermöglicht, hat sich dabei das Vorhandensein einer Implikation der Gesamtbedeutung durch die Bedeutung des Nomens erwiesen. ${ }^{66}$ Vergleicht man den hier vorgeschlagenen Neuansatz mit der traditionellen Herangehensweise, so fällt, was die Zugehörigkeit einzelner Verbindungen zur jeweiligen Klasse betrifft, vor allem ein signifikanter Unterschied auf: Kausativa (zum Lachen bringen, in Bewegung setzen usw.), die in den meisten herkömmlichen Klassifikationen zum festen Bestand der FVG gerechnet werden, sind keine FVG im hier festgelegten Sinne. ${ }^{67}$

Dass Kausativa nicht in die Klasse einbezogen werden, ist indes zu verschmerzen. Angesichts der Tatsache, dass morphologische Kausativa des Typs tränken nicht mehr produktiv sind, ist das Vorhandensein kausativer Konstruktionen wie in Bewegung setzen oder zum Lachen bringen vollkommen erwartbar.

66 Eine Implikationsrelation - allerdings zwischen Nomen und Verb und nicht, wie hier angenommen, zwischen Nomen und gesamtem Gefüge - hat übrigens bereits Bahr (1977) in ihrer insgesamt zu wenig rezipierten Dissertation als klassenkonstituierendes Merkmal aufgestellt, vgl. Bahr (1977: 260, 269). Eine Implikation zwischen Nomen und Verb ist jedoch in vielen Fällen nur schwer formulierbar, besonders wenn es sich um Verben wie zollen handelt, denen keine (bzw. keine konstruktionsexterne) Bedeutung zugeschrieben werden kann.

67 Einwände gegen die Klassifikation von Kausativa als FVG sind jedoch auch schon früher geäußert worden, etwa von Bahr (1977: 268) und besonders von Persson (1992: 156, 158). 
Zudem ist der Aufbau dieser analytischen Kausativa verhältnismäßig unauffällig: Das Verb bringen, setzen o. ä. steht für das bewirkende Ereignis, das Nomen in der PP drückt das bewirkte Ereignis aus. Die Fügung ist damit als kompositional zu bewerten. FVG im hier definierten Sinne - zumindest deren zentrale Vertreter - entsprechen dagegen weit weniger den Erwartungen an eine regelkonforme Nomen-Verb-Verbindung: Dass das Nomen einer Fügung mit tautologischem oder konversem Bedeutungsaufbau die lexikalische Bedeutung der gesamten Konstruktion enthält und das Verb keine eigene Prädikation aufweist, ist ungewöhnlich und wohl in der Tat als ein Alleinstellungsmerkmal dieser Fügungen innerhalb des Sprachsystems anzusehen. Durch die Tatsache, dass sich zahlreichen FVG - im Unterschied zu kausativen Konstruktionen wie zum Lachen bringen - ein etymologisch verwandtes Simplexverben an die Seite stellen lässt (zur Aufführung bringen - aufführen), wird zudem die Frage aufgeworfen, weshalb es in einer Sprache wie dem Deutschen überhaupt eine komplexe NomenVerb-Verbindung gibt, wenn gleichzeitig ein synonymes einfaches Verb zur Verfügung steht, das den betreffenden Inhalt offenbar ebenso gut ausdrücken kann. Das Vorhandensein von FVG ist damit nicht allein ein Problem der Sprachkritik, sondern fordert auch die Sprachtheorie zu einer Antwort heraus: Immerhin stellt eine weitgehende Synonymie einfaches Verb/FVG die Grundannahme der Ökonomie des Lexikons in Frage: „Warum leistet sich aber eine Sprache eine derart große Zahl ,semantischer Dubletten“ [...]?“ (Storrer 2006: 148). Eine Kategorie FVG, die sich auf diese erklärungsbedürftigen Fälle konzentriert, bietet insgesamt eine deutlich bessere Ausgangslage für die Lösung der angesprochenen Fragen als die herkömmliche Klasse FVG, die Problematisches und Unproblematisches miteinander vermischt.

$\mathrm{Zu}$ verschmerzen ist sicher auch, dass durch das Entfallen der kausativen Fügungen in einigen Fällen die Reihenbildung - in Bewegung setzen/kommen/sein usw. - um ein Element reduziert wird: Kausatives in Bewegung setzen wäre kein FVG, in Bewegung sein bzw. kommen wären immerhin periphere Vertreter der Kategorie FVG. Die Bildung von Oppositionsreihen ist in der Forschung zwar immer wieder als wesentliches Charakteristikum von FVG herausgestellt worden, es hat sich aber gezeigt, dass solche Reihenbildungen weder für alle FVG gelten noch ausschließlich bei FVG vorkommen (s. Kapitel 2.2.4.2). Mit der Oppositionsbildung entfällt somit ein ohnehin nur sehr schwaches Klassifikationskriterium. Dieser Befund wird dadurch unterstrichen, dass Oppositionen zweier oder mehrerer Ausdrücke auch in anderen Bereichen keine gemeinsame Klassenzugehörigkeit erzwingen: Die semantische Opposition (Konverse) zwischen den Verben mieten und vermieten bzw. kaufen und verkaufen etwa ändert nichts daran, dass 
diese Verben hinsichtlich ihrer Argumentstruktur unterschiedlichen Klassen Transitiv und Ditransitiv - angehören.

Der wesentliche Vorteil der Klasse ,implikative Verbindungen` gegenüber der herkömmlichen Kategorie FVG besteht somit - so lässt sich zusammenfassen - darin, dass mit der Implikation Nominalbedeutung $\rightarrow$ Gesamtbedeutung ein zentrales klassendefinierendes Kriterium genannt werden kann, das bei allen Vertretern der Kategorie und auch nur bei diesen vorliegt. Damit ist eine deutlich bessere Abgrenzung der Klasse nach außen erreicht. Das Vorhandensein eines und nur eines - definierenden Kriteriums macht die Entscheidung, welche Nomen-Verb-Verbindung zu Klasse gehört und welche nicht, wesentlich besser überprüfbar als das heterogene Set von Kriterien, das die FVG-Forschung bisher herausgearbeitet hat. Deren Kriterien - Aktionsart, prädikativer Eigenbeitrag, Reihenbildung, semantisch „leeres“ Verb usw. - konnten allenfalls für einzelne Gruppen von FVG, nie aber für die gesamte Klasse Geltung beanspruchen.

Ein weiterer Vorteil der vorgeschlagenen Definition ist darin zu sehen, dass die Klasse, der das Nomen in FVG angehört, klar abgegrenzt ist: Es kommen nur relationale Nomina vor, d. h. Nomina, die ein Eigenschaftskonzept im Sinne von Abschnitt 3.2.1 kodieren. Dies ergibt sich unmittelbar aus der Definition Nominalbedeutung $\rightarrow$ Gesamtbedeutung. Da die Gesamtbedeutung eines FVG wegen seines verbalen Kopfes stets relational ist, muss auch die Nominalbedeutung relational sein; andernfalls wäre keine Implikationsrelation formulierbar. Damit sind umstrittene Fälle wie jmdn. auf die Palme bringen oder auch etwas zur Sprache, jmdn. zur Vernunft bringen (van Pottelberge 2001: 325, 455) von vornherein ausgeschlossen. In diesem Punkt ist der FVG-Begriff dieser Untersuchung mit der Klassenbestimmung von Fleischer (1997) vergleichbar, der die Nomina in FVG im Wesentlichen auf Ereignisnominalisierungen beschränkt hatte. Während bei Fleischer jedoch unklar geblieben war, weshalb eine solche Einschränkung nötig sein sollte, ergibt sich diese hier direkt aus der zugrundegelegten Definition.

Zwar ist das hier in den Mittelpunkt gestellte Kriterium der Implikation Nominalbedeutung $\rightarrow$ Gesamtbedeutung in unterschiedlich starken Ausprägungen gegeben. Der Abgrenzbarkeit der implikativen Syntagmen gegenüber anderen Typen von Nomen-Verb-Verbindungen tut dies jedoch keinen Abbruch. Die Gradierbarkeit des hier gewählten Definitionskriteriums macht auch deutlich, dass manche FVG im Hinblick auf ihr Verhältnis zur freien Syntax eher als regulär, andere als eher irregulär zu bewerten sind: Gegenüber den tautologischen und konversen FVG verhalten sich kursive und inchoative FVG tendenziell eher regelkonform, da dem Verb hier, wie erwähnt, eine Bedeutung zugesprochen werden kann, während das Verb tautologischer und konverser Fügungen nicht Träger ei- 
ner eigenen lexikalischen Bedeutung ist. Die noch folgenden Kapitel dieser Untersuchung werden sich deshalb ganz überwiegend den konversen und tautologischen und damit den eher irregulär erscheinenden FVG als den prototypischen Vertretern der Kategorie annehmen und inchoative sowie imperfektive NomenVerb-Verbindungen nur am Rande behandeln. Die prototypischen FVG werfen für sich genommen jedenfalls genug Fragen auf, wie sich im Weiteren zeigen wird. 